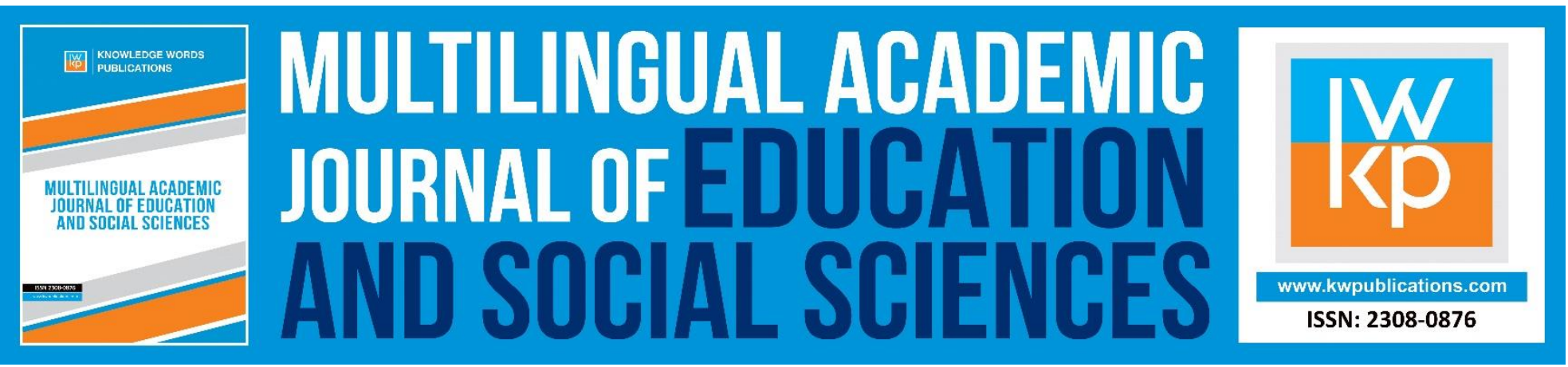

\title{
Toddler Engagement in Digital Aloud Reading: The Role of Variations in Designing and Presentation of Digital Stories
}

\author{
Eleni Gkantia \& Kostas D. Dinas
}

To Link this Article: http://dx.doi.org/10.46886/MAJESS/v8-i1/7275

DOI: 10.46886/MAJESS/v8-i1/7275

Received: 08 April 2020, Revised: 09 May 2020, Accepted: 20 June 2020

Published Online: 27 July 2020

In-Text Citation: (Gkantia \& Dinas, 2020)

To Cite this Article: Gkantia, E., \& Dinas, K. D. (2020). Toddler Engagement in Digital Aloud Reading: The Role of Variations in Designing and Presentation of Digital Stories. Multilingual Academic Journal of Education and Social Sciences, 8(1), 151-167.

\section{Copyright: (c) The Authors 2020}

Published by Knowledge Words Publications (www.kwpublications.com)

This article is published under the Creative Commons Attribution (CC BY 4.0) license. Anyone may reproduce, distribute, translate and create derivative works of this article (for both commercial and non-commercial purposes), subject to full attribution to the original publication and authors. The full terms of this license may be seen

at: http://creativecommons.org/licences/by/4.0/legalcode

Vol. 8, No. 1, 2020, Pg. 151 - 167

https://kwpublications.com/journals/journaldetail/MAJESS

JOURNAL HOMEPAGE

Full Terms \& Conditions of access and use can be found at https://kwpublications.com/pages/detail/publication-ethics 


\title{
Toddler Engagement in Digital Aloud Reading: The Role of Variations in Designing and Presentation of Digital Stories
}

\author{
Eleni Gkantia \& Kostas D. Dinas \\ Department of Early Childhood Education, University of Western Macedonia, Florina, Greece \\ Email: gantiaeleni@gmail.com,kdinas@uowm.gr
}

\begin{abstract}
The aim of this study is to examine the influence of design and presentation characteristics of digital stories in the engagement of toddlers in digital aloud reading. The sample was comprised of 26 children of kindergarten and pre-kindergarten age, who, after having been separated in three groups, were presented with the same digital aloud reading, but different variations for each group: interactive presentation, cartoons, static images and oral storytelling. Multi-sensory behaviors and the communication behavior were videotaped during the digital read aloud. The data were aggregated per child in one-minute intervals, examining their simultaneous behavior and they were then consolidated in order to determine the frequency measures of individual toddler behavior per digital read aloud variation/type. The results show that the type influences an array of engagement behaviors in digital read aloud. Looking, touching, moving and gesturing were all significantly different depending on the presentation type of the digital story, whereas no major differences were observed as regards communication behavior.
\end{abstract}

Keywords: Digital Read Aloud, Engagement, Characteristics in The Design and Presentation of Digital Stories.

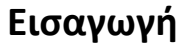

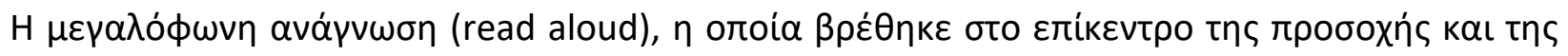

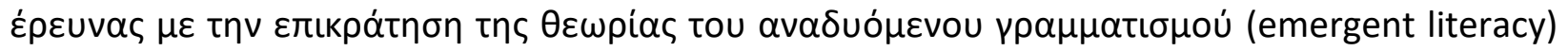

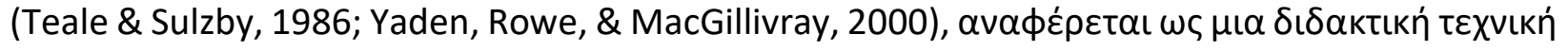

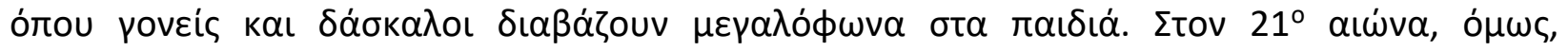

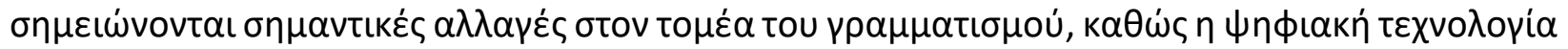

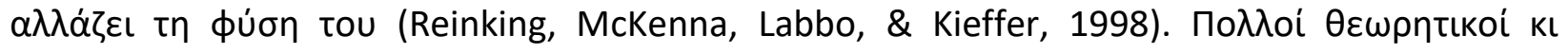

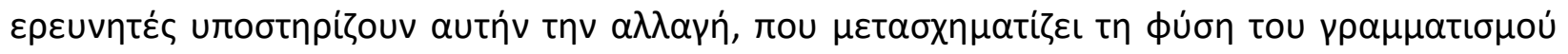

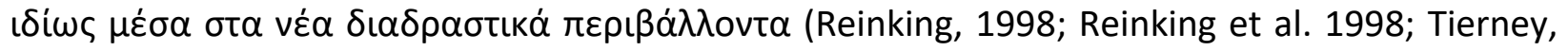

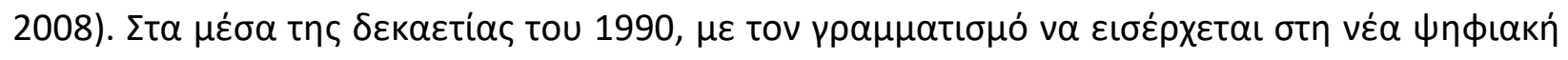


MULTILINGUAL ACADEMIC JOURNAL OF EDUCATION AND SOCIAL SCIENCES

Vol. 8 No. 1, 2020, E-ISSN: 2308-0876 @ 2020 KWP

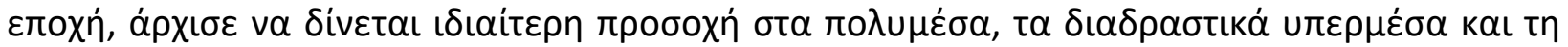

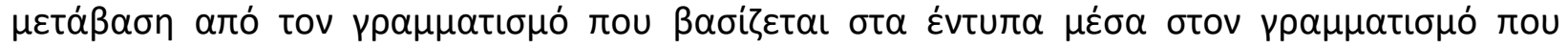

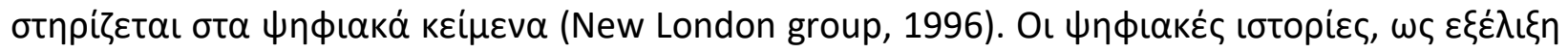

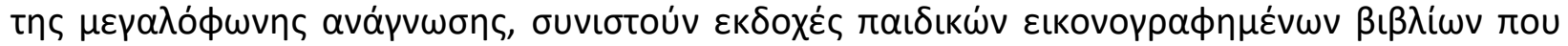

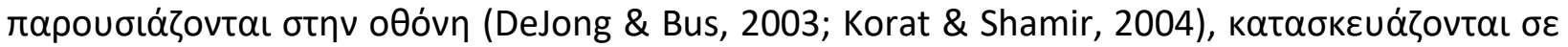

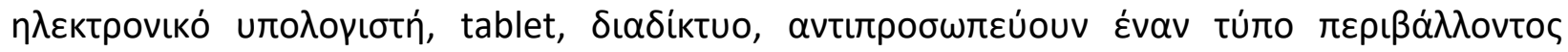

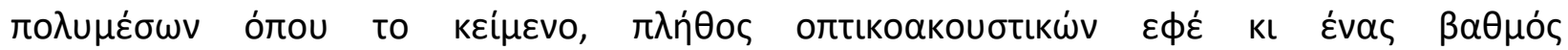

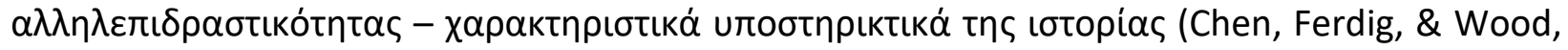

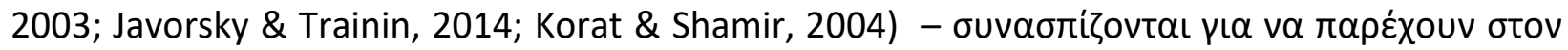

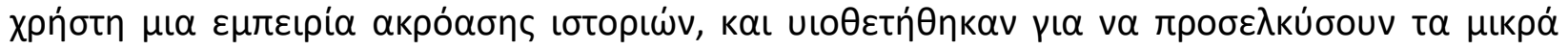

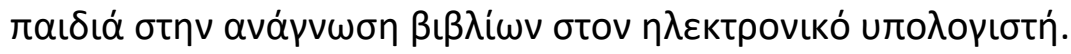

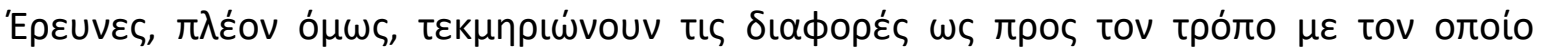

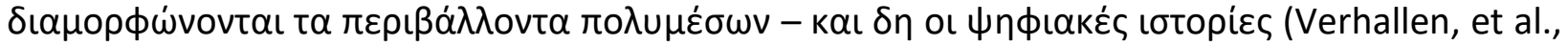

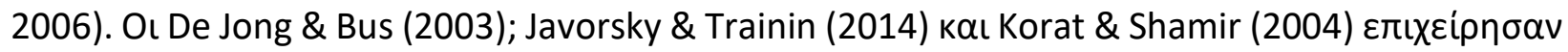

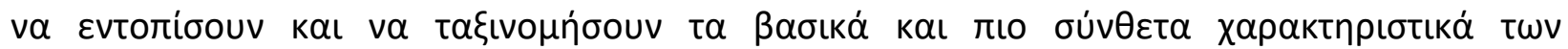

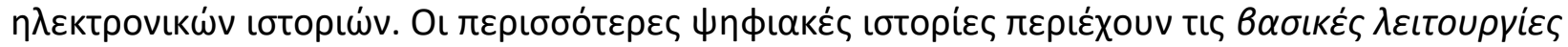

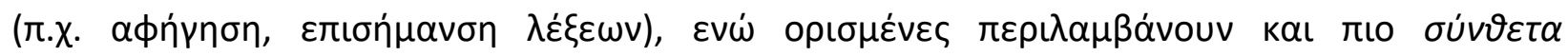

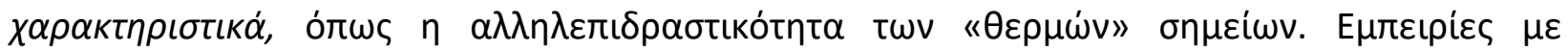

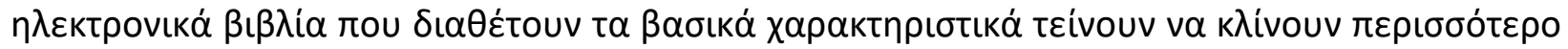

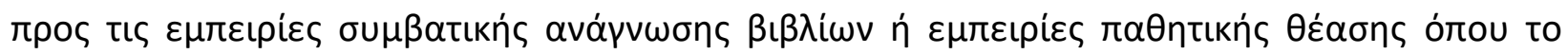

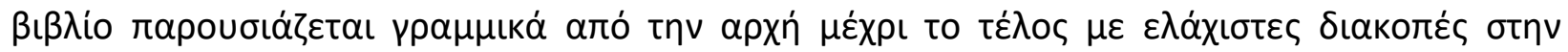

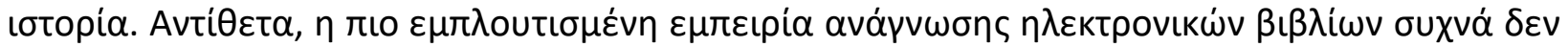

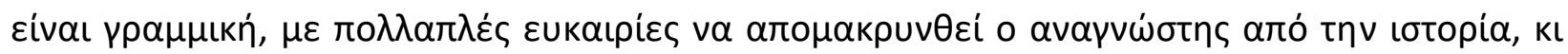

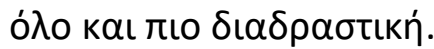

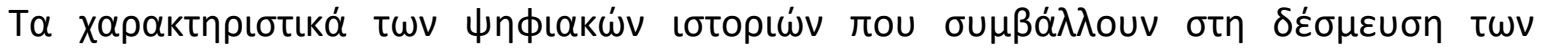

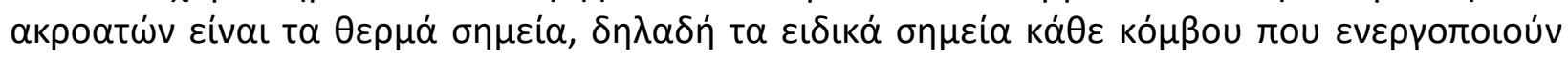

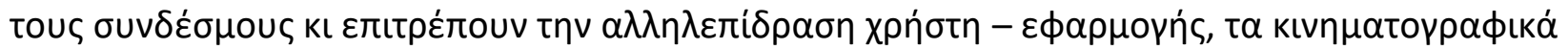

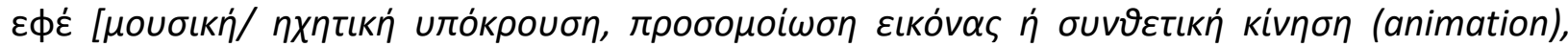

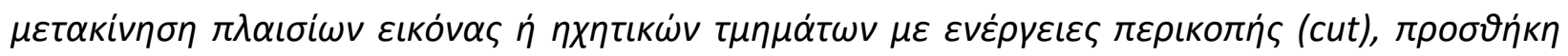

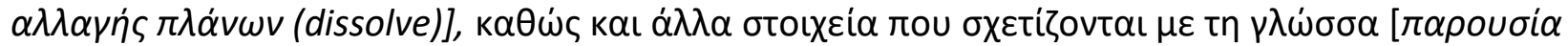

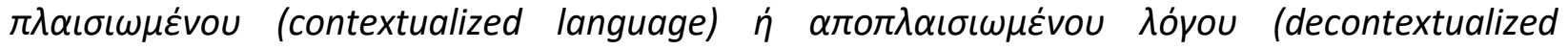

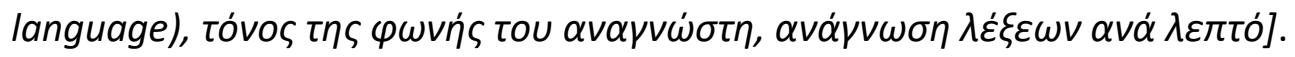

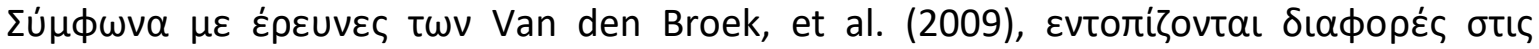

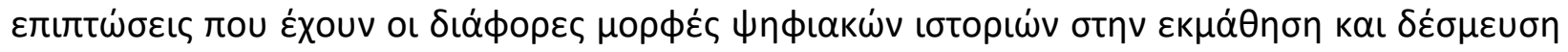

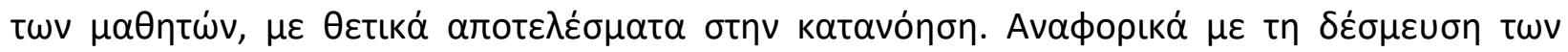

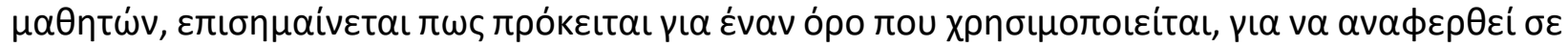

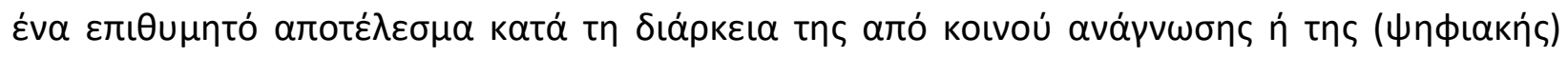

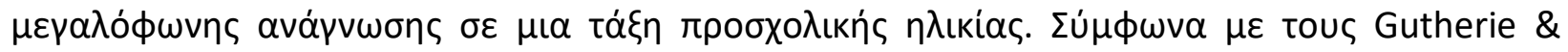

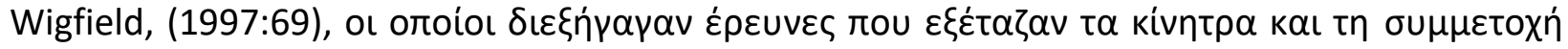

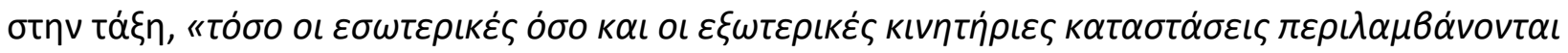

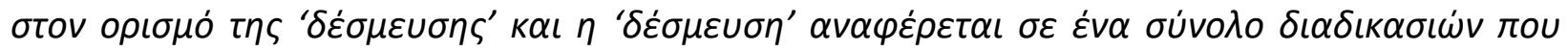

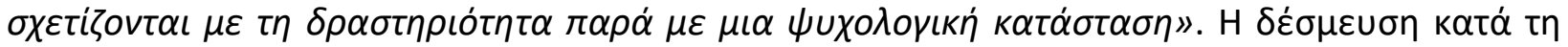

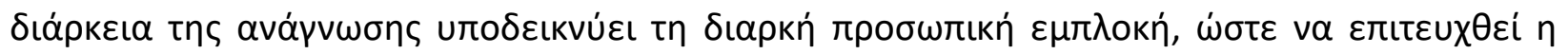


MULTILINGUAL ACADEMIC JOURNAL OF EDUCATION AND SOCIAL SCIENCES

Vol. 8 No. 1, 2020, E-ISSN: 2308-0876 @ 2020 KWP

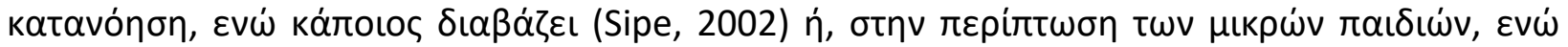

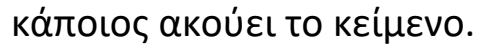

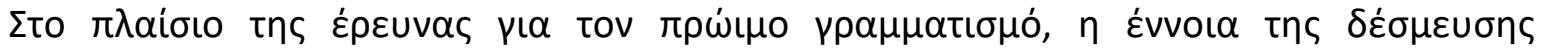

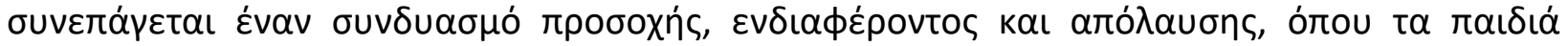

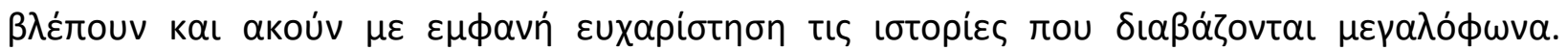

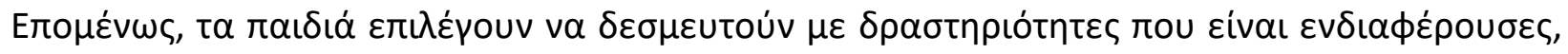

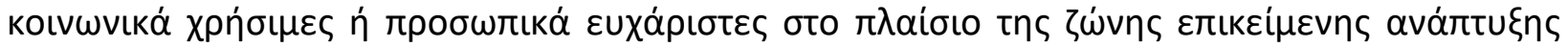

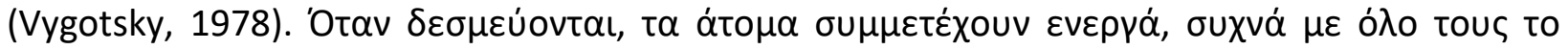

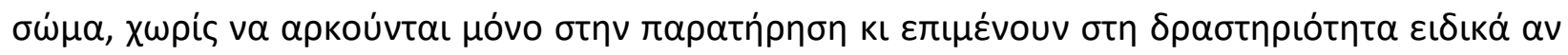

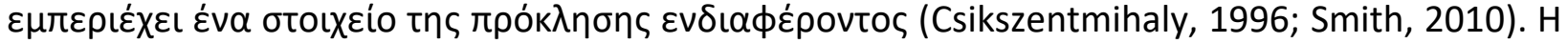

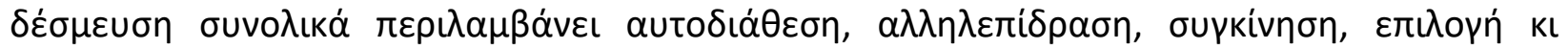

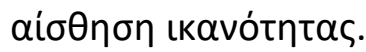

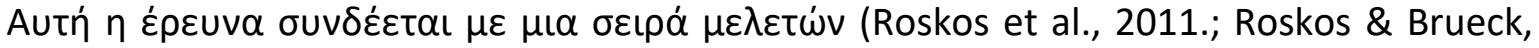

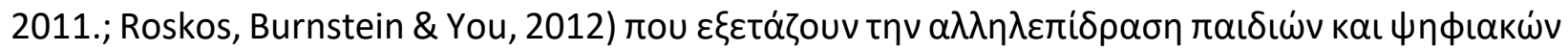

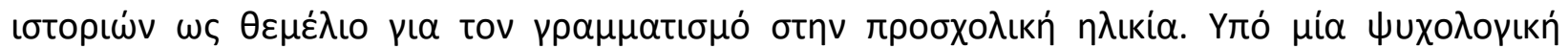

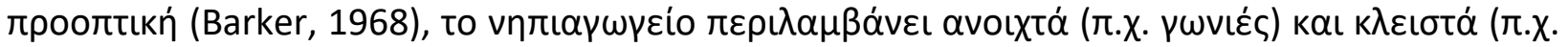

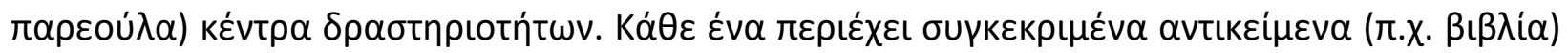

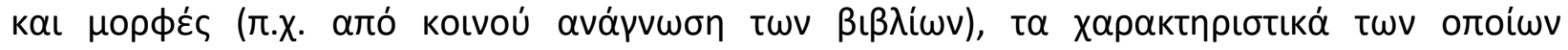

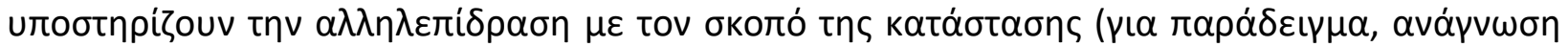

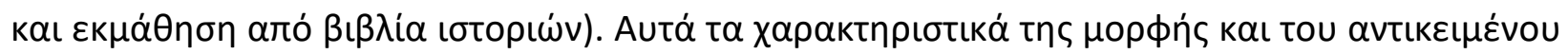

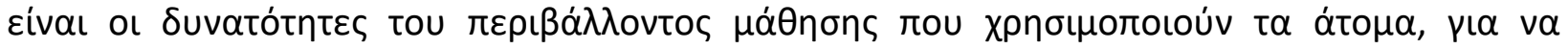

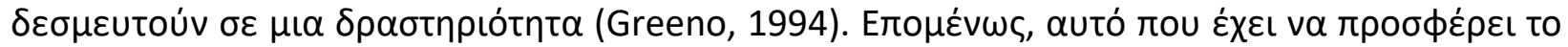

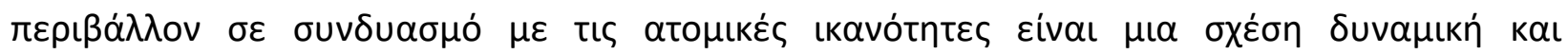

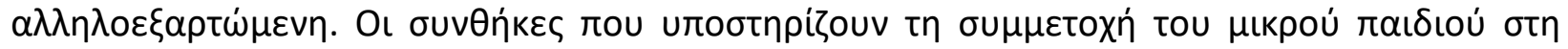

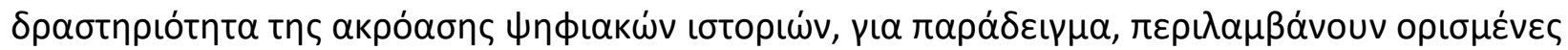

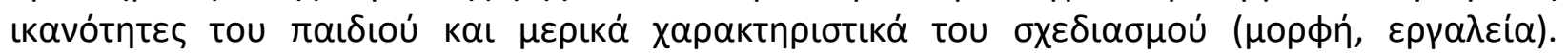

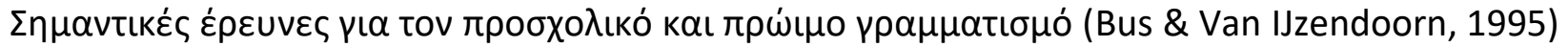

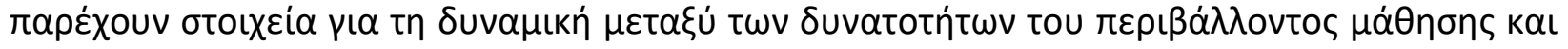

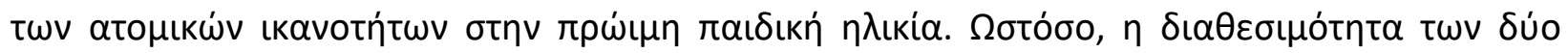

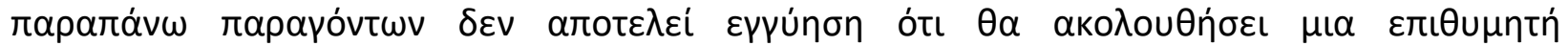

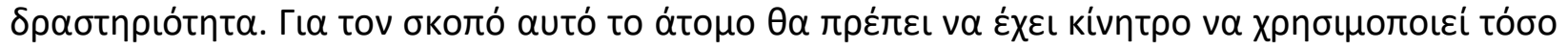

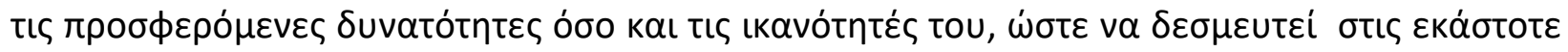

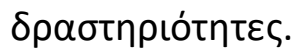

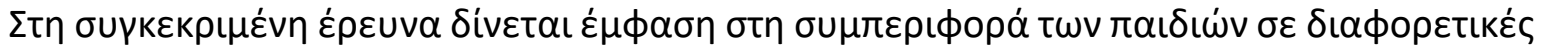

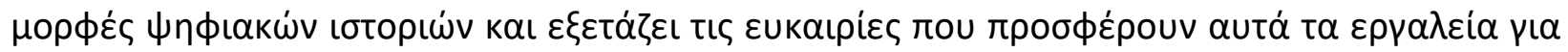

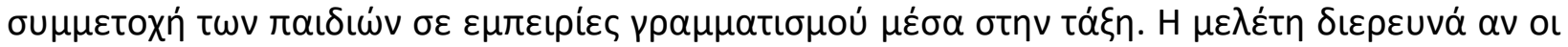

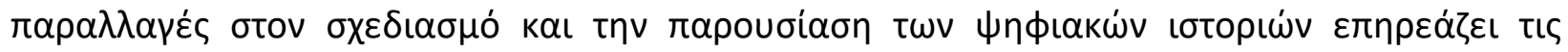

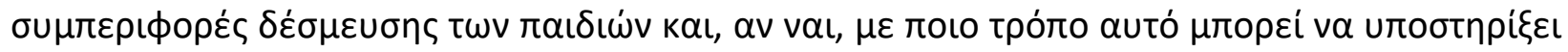

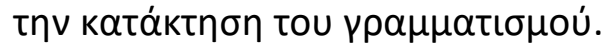

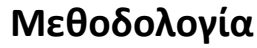

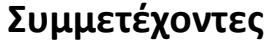

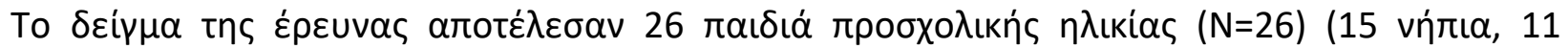

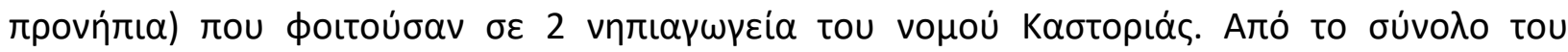


MULTILINGUAL ACADEMIC JOURNAL OF EDUCATION AND SOCIAL SCIENCES

Vol. 8 No. 1, 2020, E-ISSN: 2308-0876 @ 2020 KWP

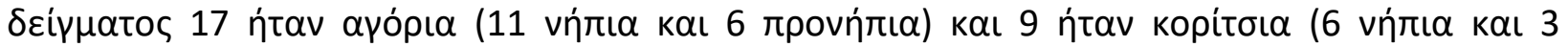

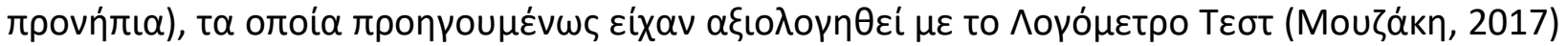

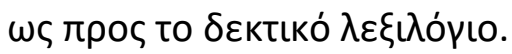

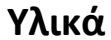

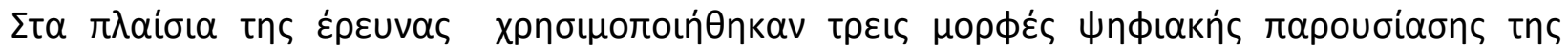

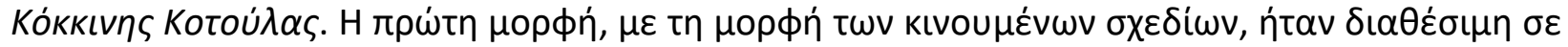

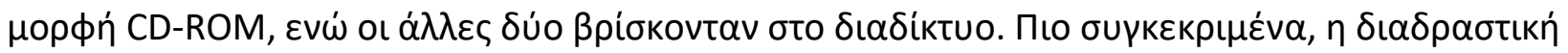

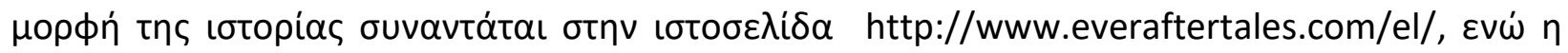

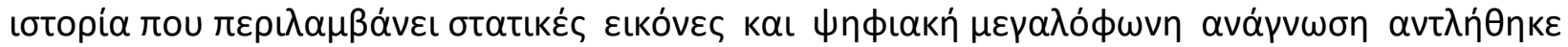

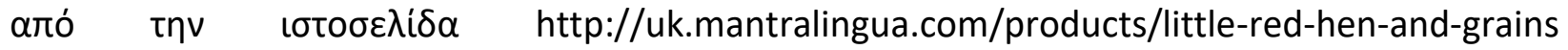

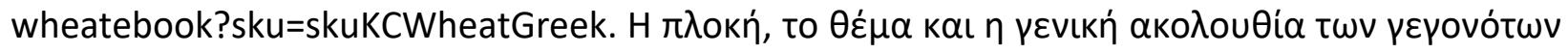

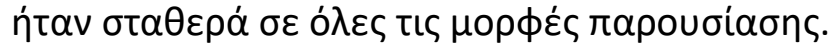

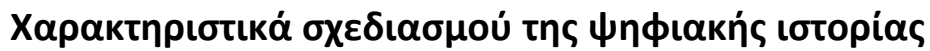

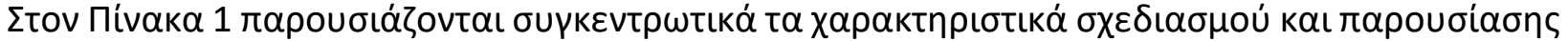

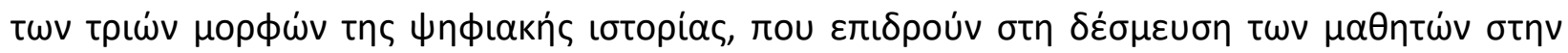

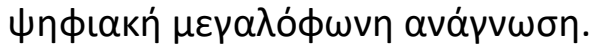

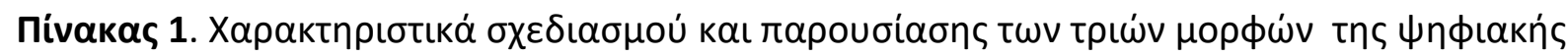
toтopías

\begin{tabular}{|c|c|c|c|}
\hline & 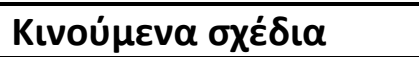 & 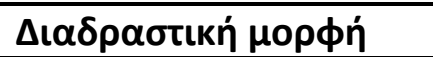 & 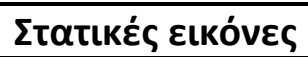 \\
\hline 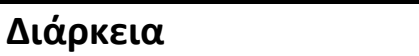 & $10: 48$ & $\begin{array}{lll}\Delta \varepsilon v & \mu \pi о \rho \varepsilon \dot{~} & v \alpha\end{array}$ & $9: 26$ \\
\hline 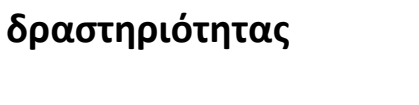 & & $\begin{array}{l}\pi \rho \circ \sigma \delta ı \rho เ \sigma \tau \varepsilon \dot{1} \quad(\alpha \lambda \lambda \alpha \dot{\alpha} \\
\pi \varepsilon ́ \rho \alpha v \tau \omega v 10 \lambda \varepsilon \pi \tau \omega \dot{v})\end{array}$ & \\
\hline 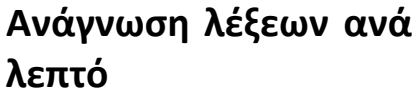 & 69 & 58 & 88 \\
\hline 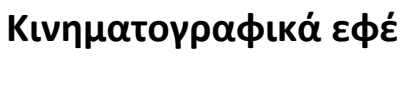 & 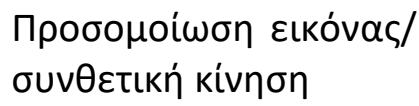 & 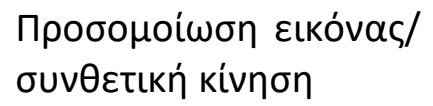 & 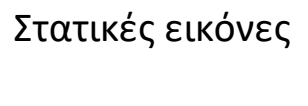 \\
\hline 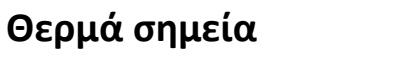 & $\Delta \varepsilon v$ uाápXouv & Eıкоvoүра́фпоп & 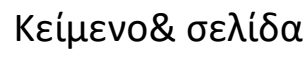 \\
\hline 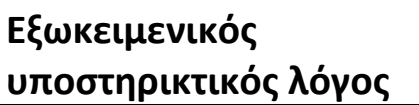 & Autó $\mu \alpha \tau \alpha$ & $M \varepsilon \ll k \lambda \iota k »$ & 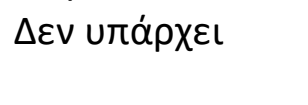 \\
\hline
\end{tabular}

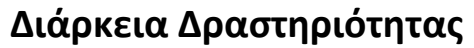

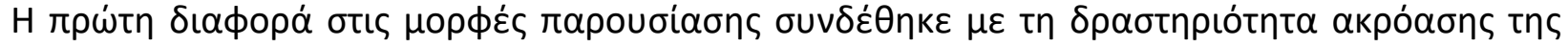

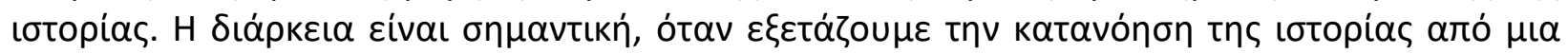

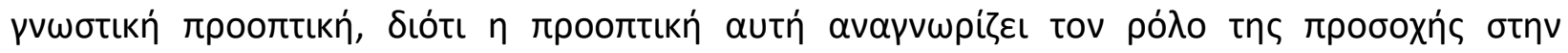

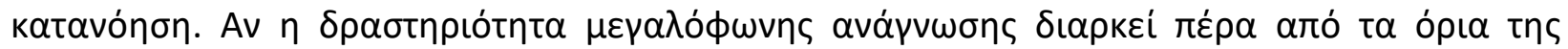

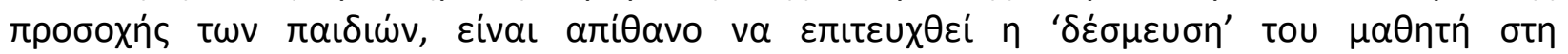

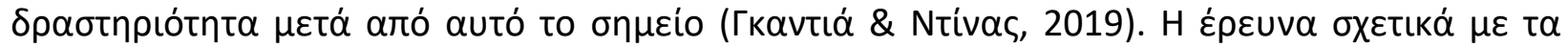

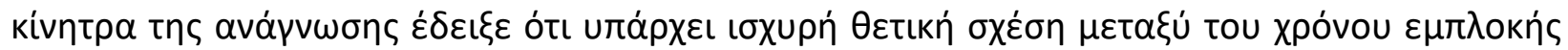

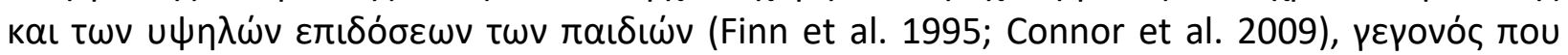

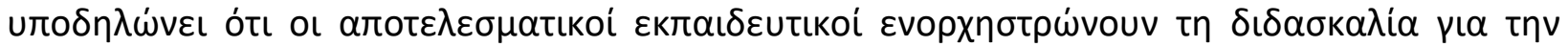

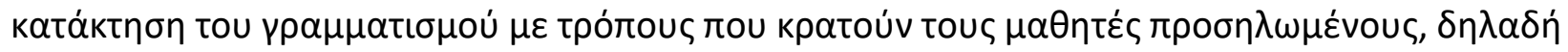


MULTILINGUAL ACADEMIC JOURNAL OF EDUCATION AND SOCIAL SCIENCES

Vol. 8 No. 1, 2020, E-ISSN: 2308-0876 @ 2020 KWP

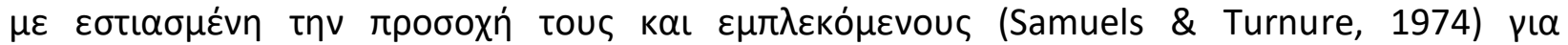

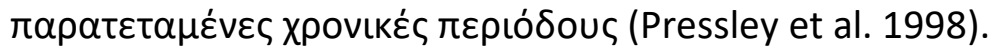

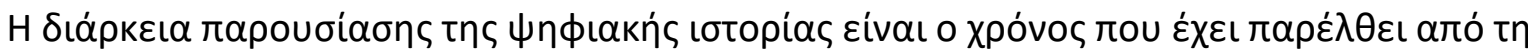

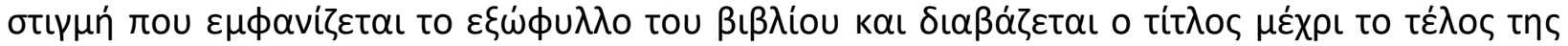

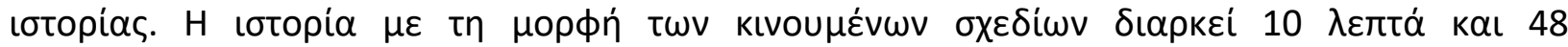

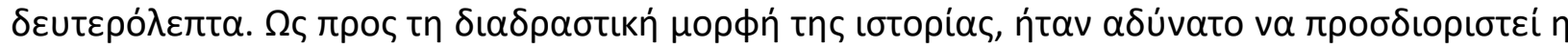

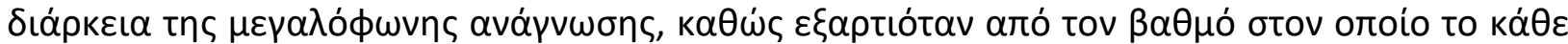

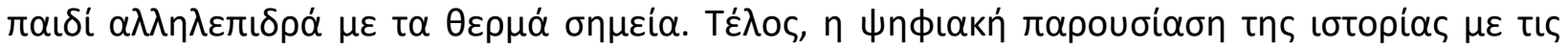

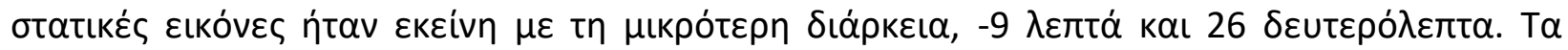

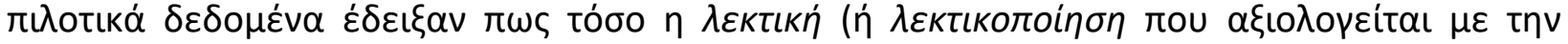

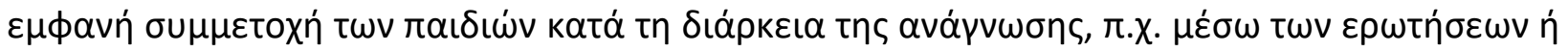

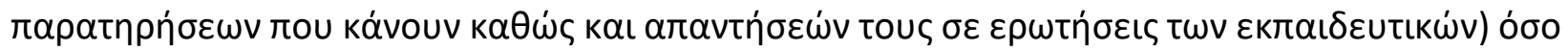

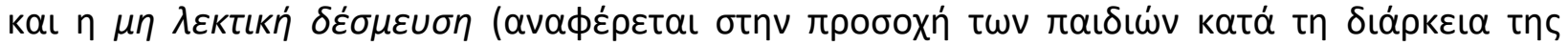

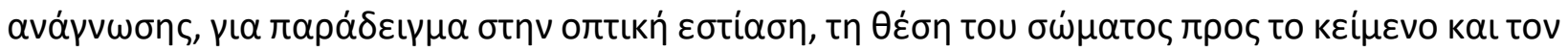

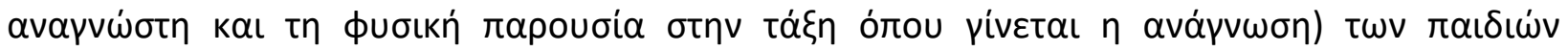

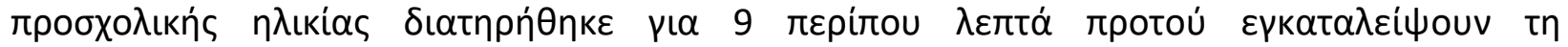

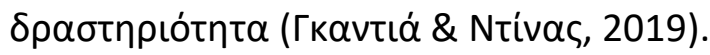

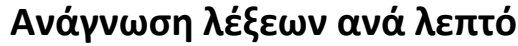

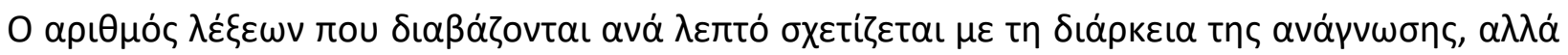

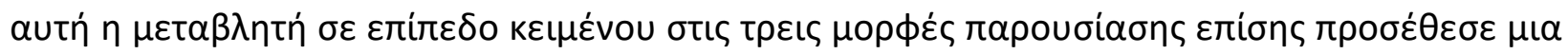

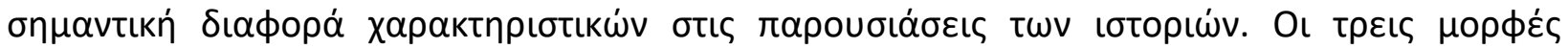

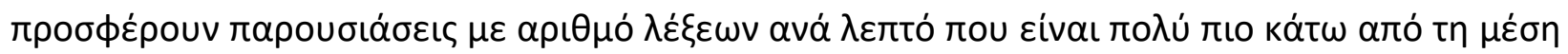

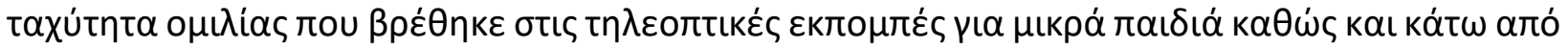

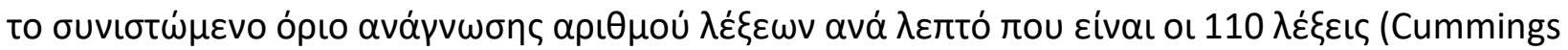

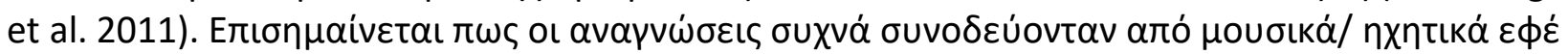

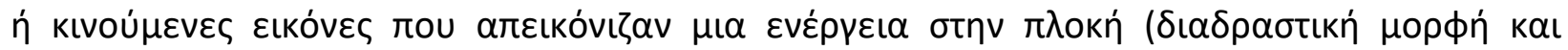

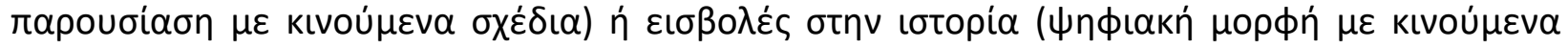

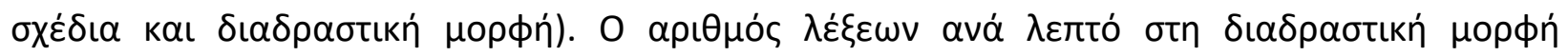

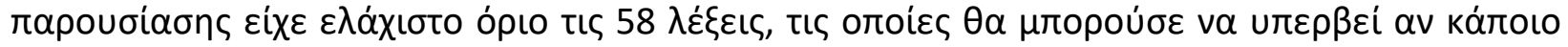

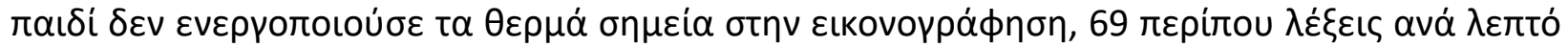

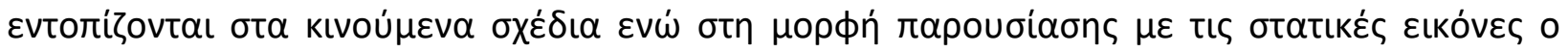

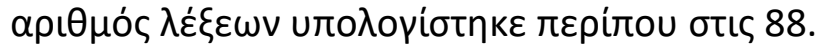

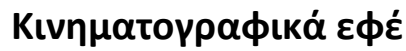

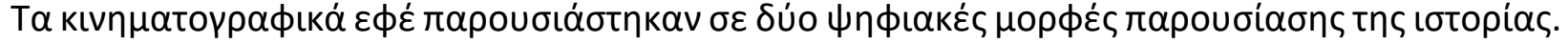

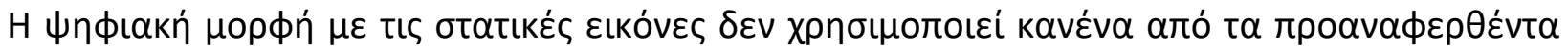

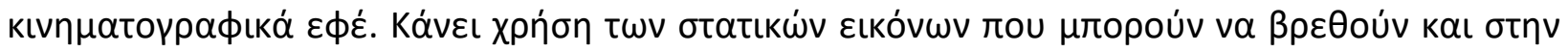

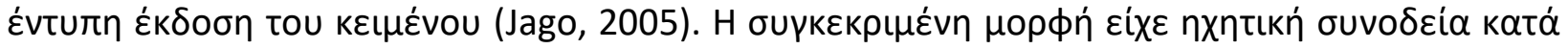

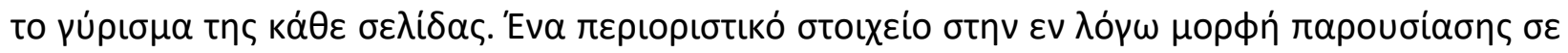

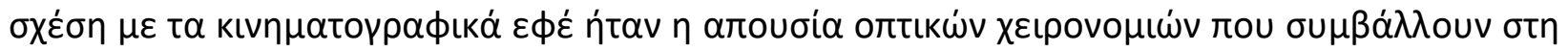

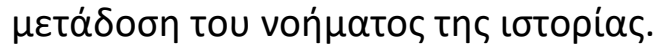

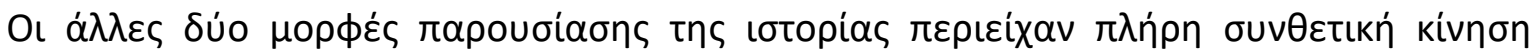

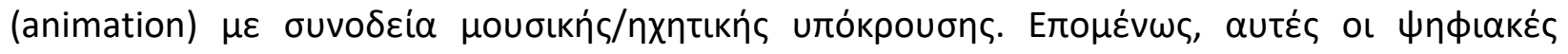




\section{MULTILINGUAL ACADEMIC JOURNAL OF EDUCATION AND SOCIAL SCIENCES \\ Vol. 8 No. 1, 2020, E-ISSN: 2308-0876 @ 2020 KWP}

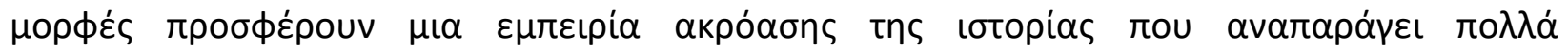

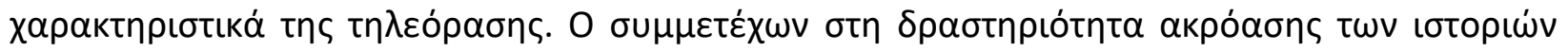

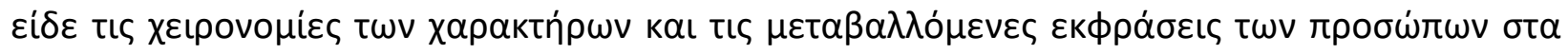

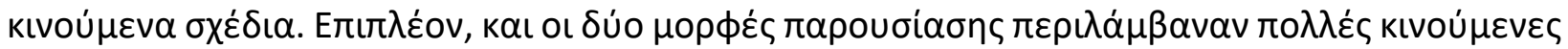

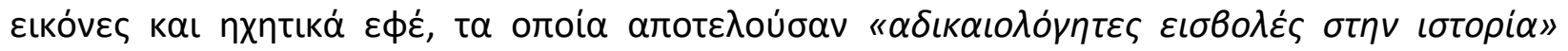

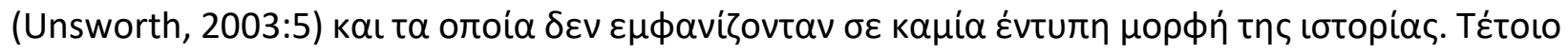

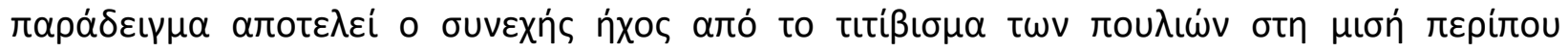

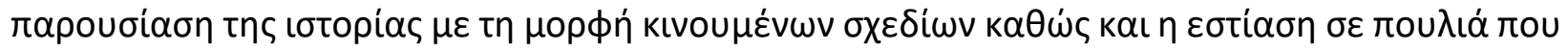

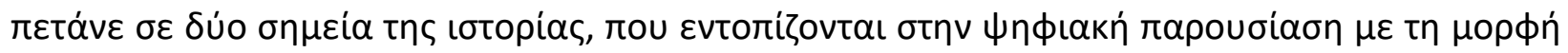

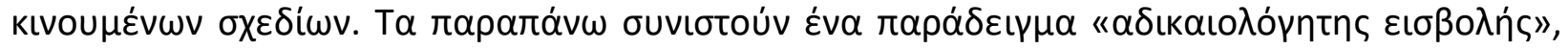

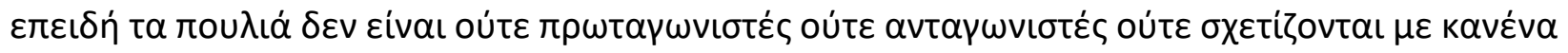

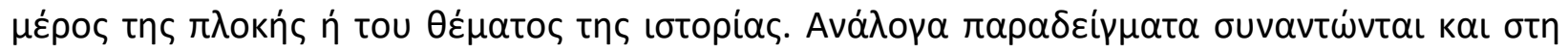

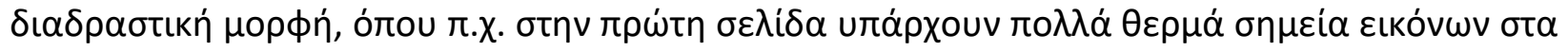

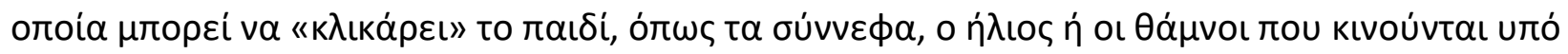

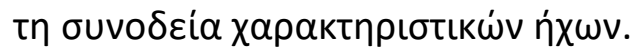

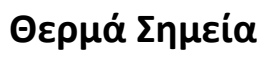

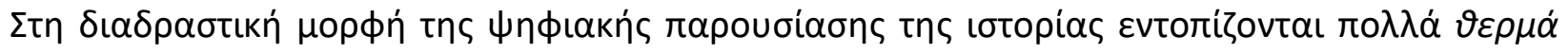

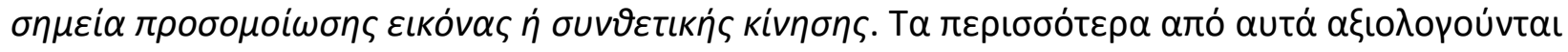

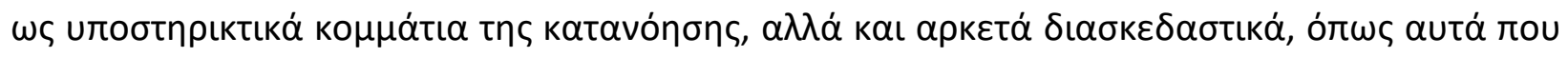

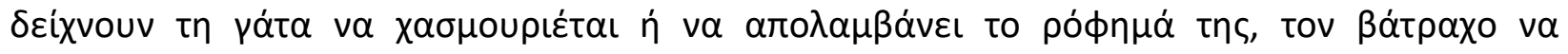

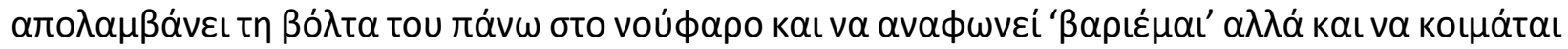

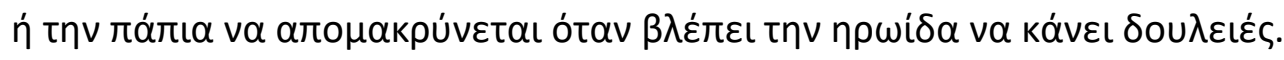

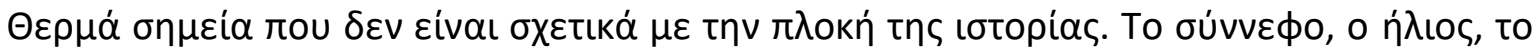

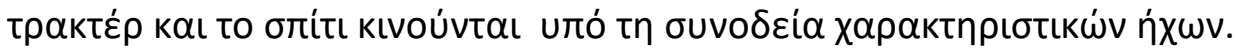




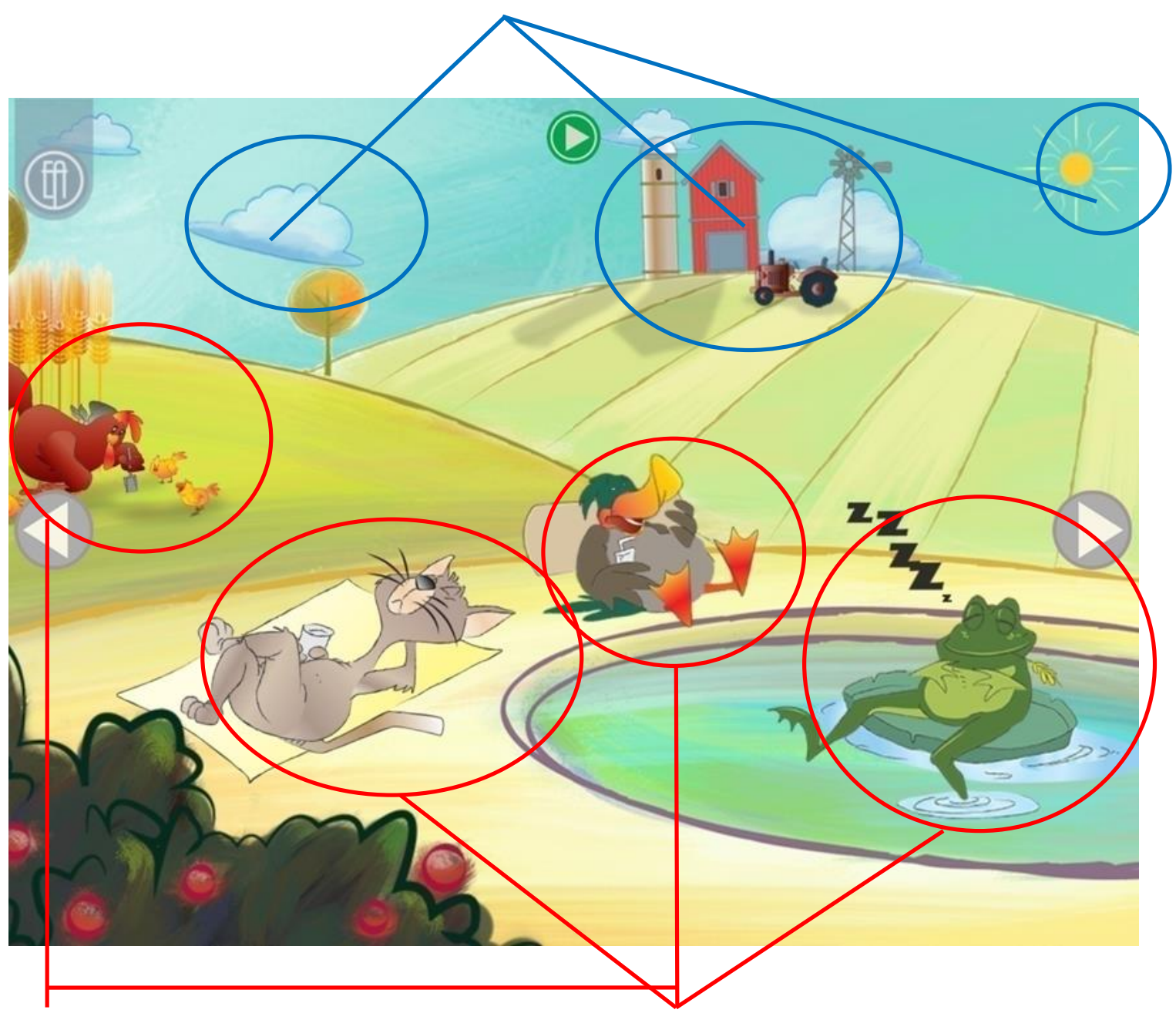

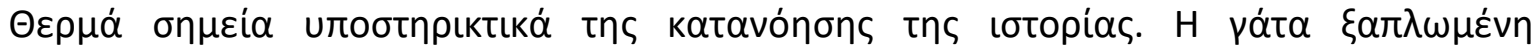

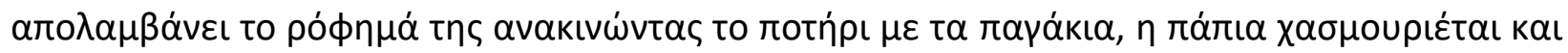

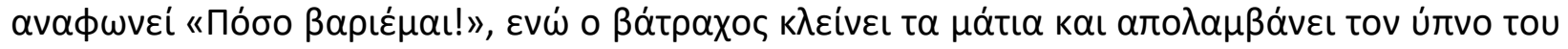

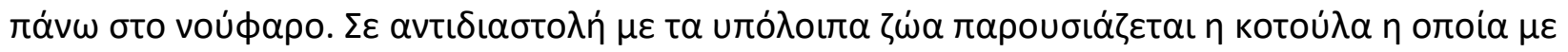

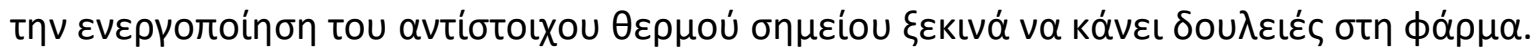

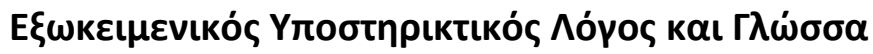

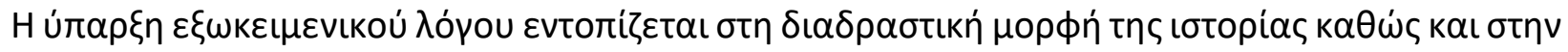

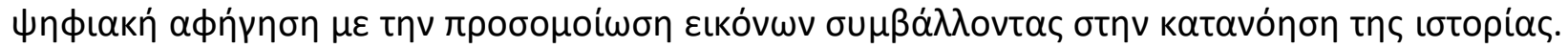

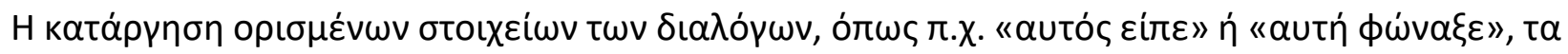

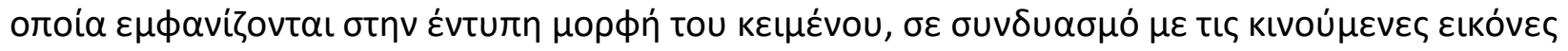

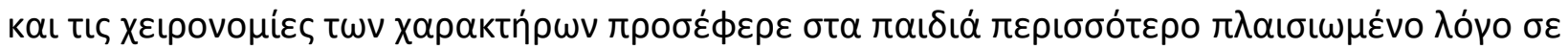

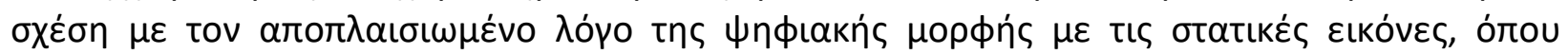

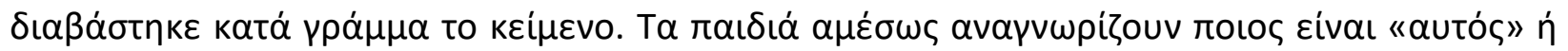

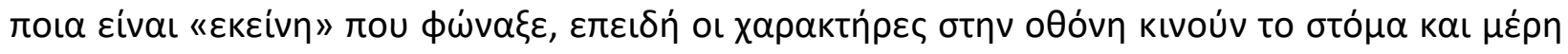

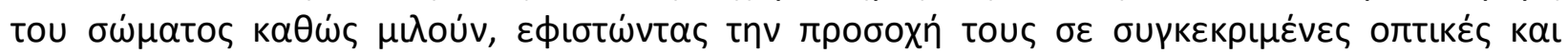

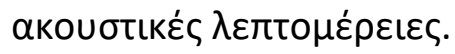


MULTILINGUAL ACADEMIC JOURNAL OF EDUCATION AND SOCIAL SCIENCES

Vol. 8 No. 1, 2020, E-ISSN: 2308-0876 @ 2020 KWP

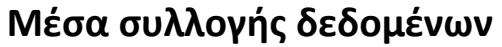

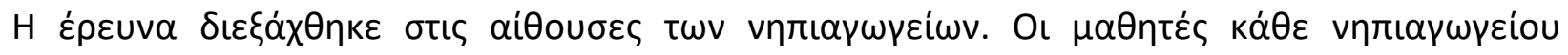

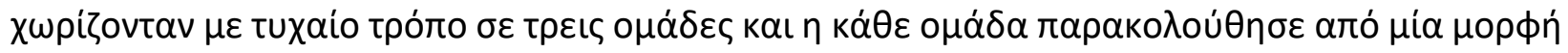

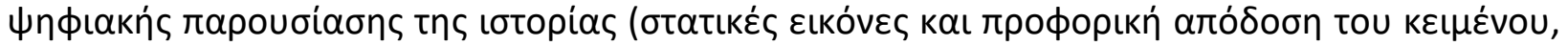

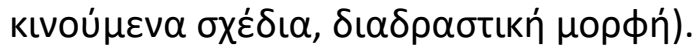

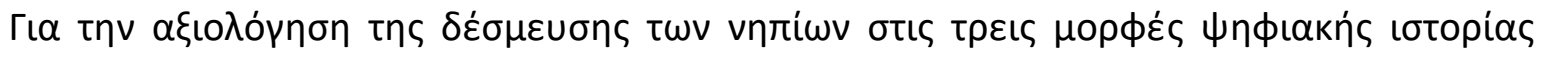

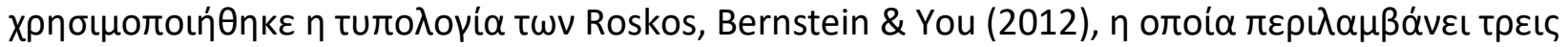

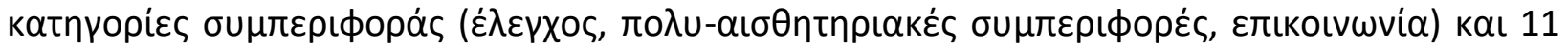

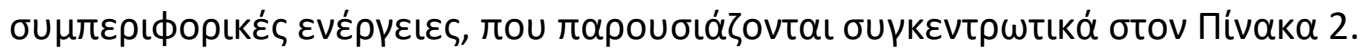

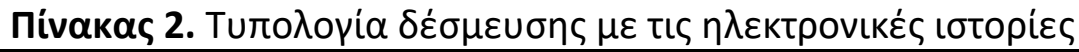

\begin{tabular}{|c|c|c|c|}
\hline \multicolumn{2}{|l|}{ Katnyopía } & \multicolumn{2}{|c|}{ 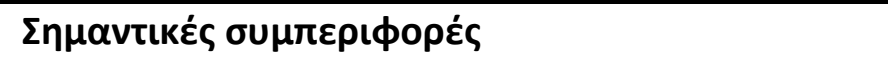 } \\
\hline 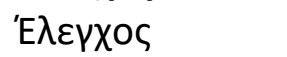 & & \multicolumn{2}{|c|}{ 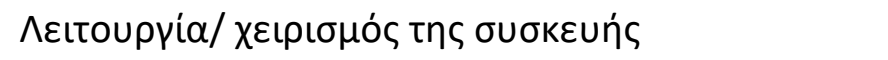 } \\
\hline \multicolumn{2}{|c|}{ 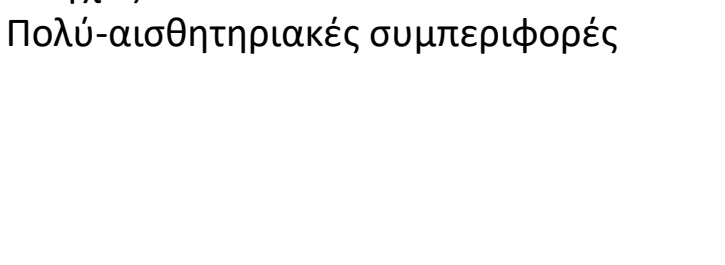 } & \multicolumn{2}{|c|}{ 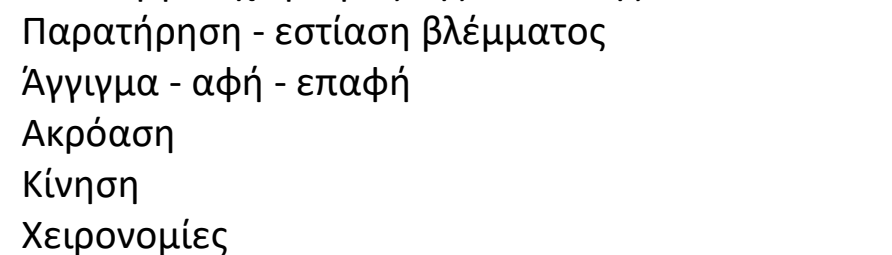 } \\
\hline \multicolumn{2}{|l|}{ 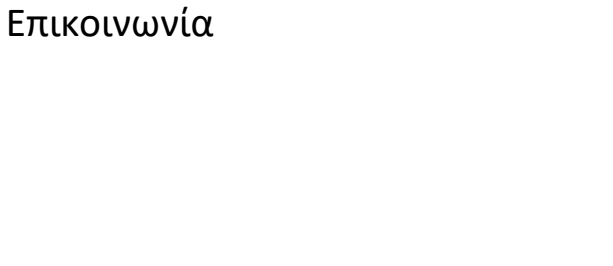 } & \multicolumn{2}{|c|}{ 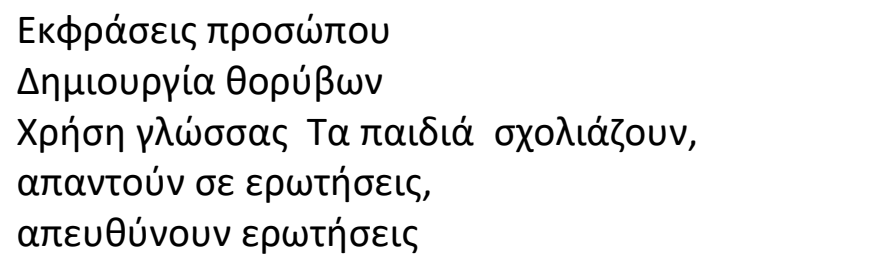 } \\
\hline 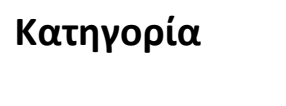 & Opıouós & 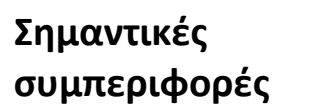 & 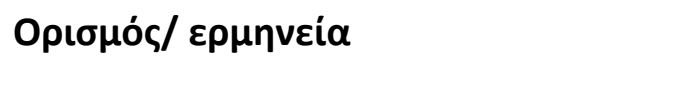 \\
\hline 'E $\lambda \varepsilon Y X O S$ & 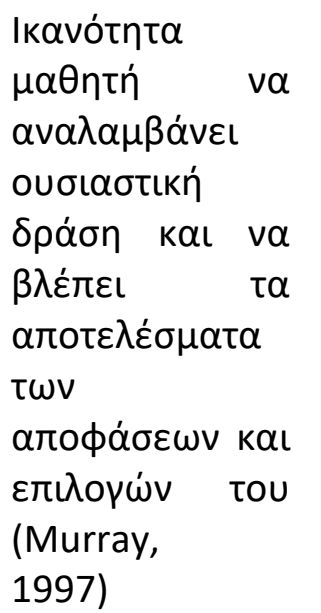 & 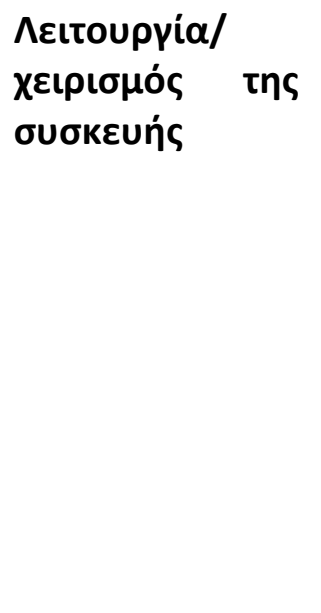 & 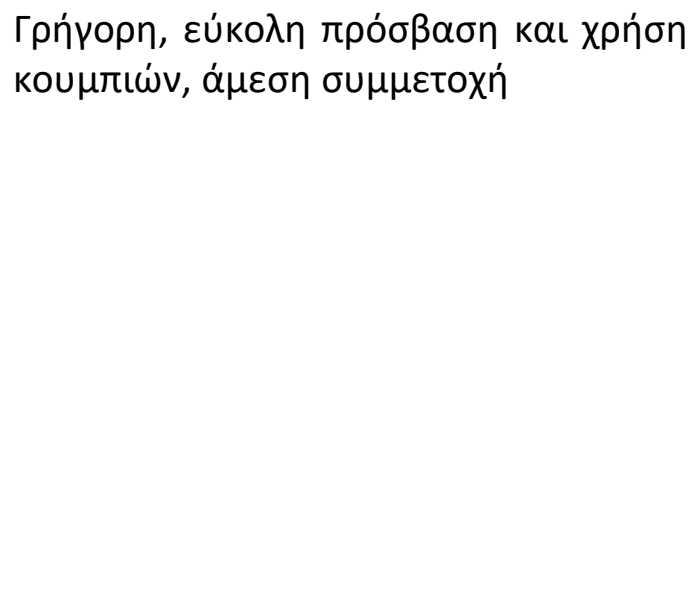 \\
\hline 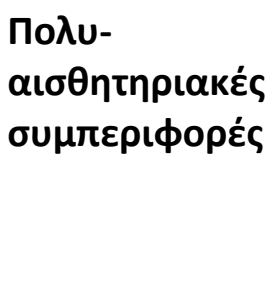 & 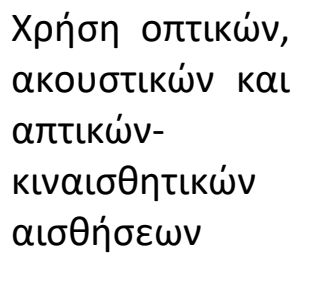 & 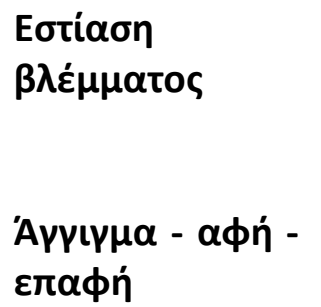 & 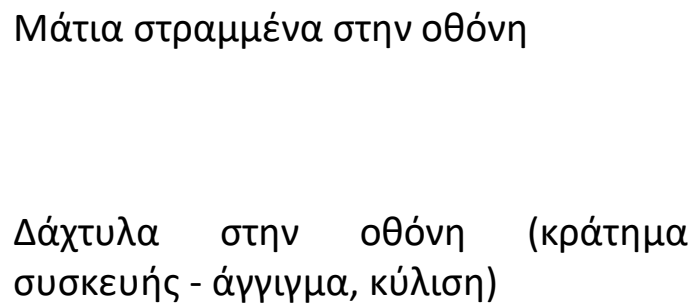 \\
\hline
\end{tabular}




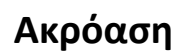

Kivnon

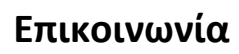

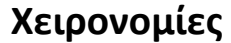

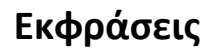
$\pi \rho \circ \sigma \dot{\pi} \pi 0 u$

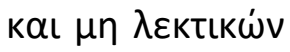

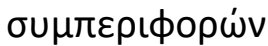

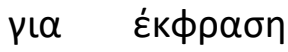
каtavónons

\section{$\Delta \eta \mu$ ioupyía Өopú $\beta \omega v$}

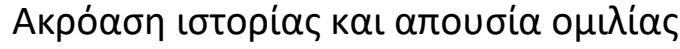

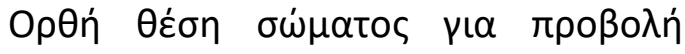
เotopias

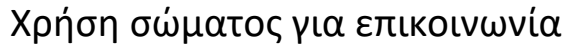

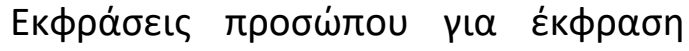

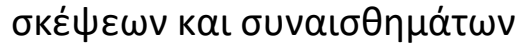

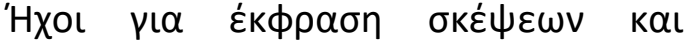

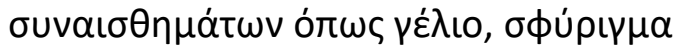

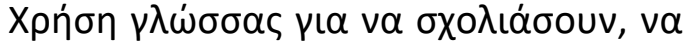

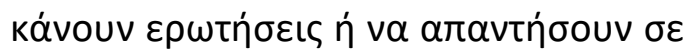

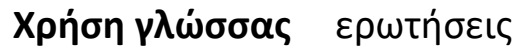

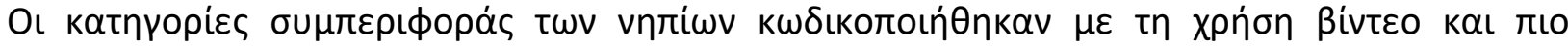

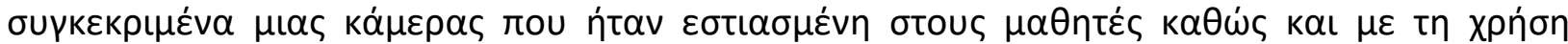

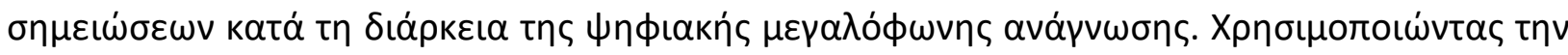

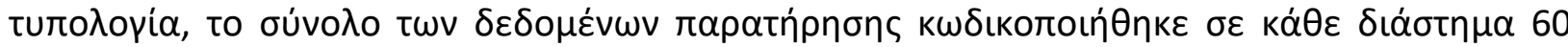

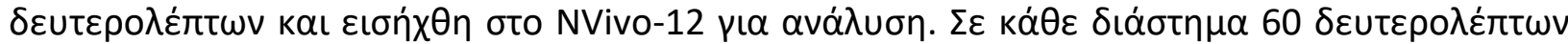

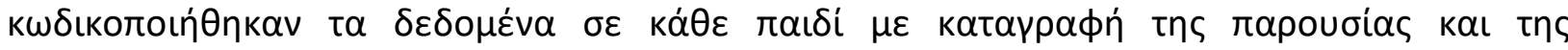

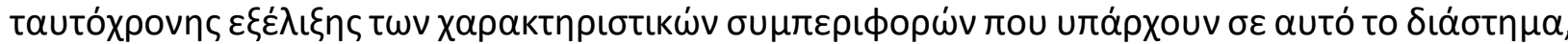

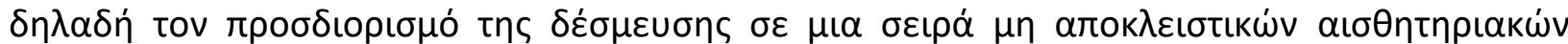

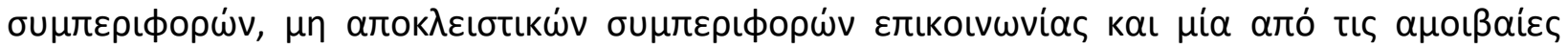

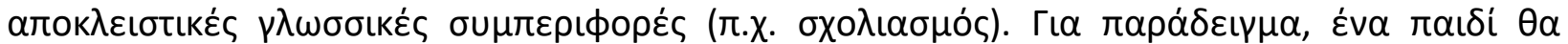

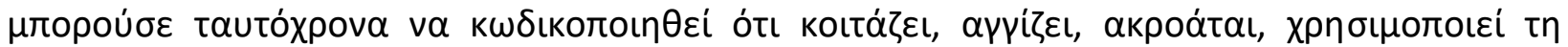

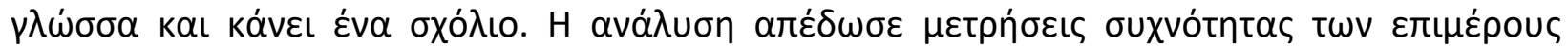

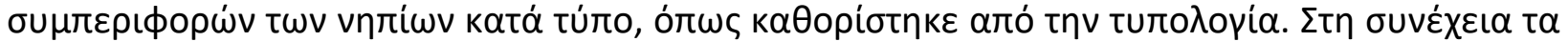

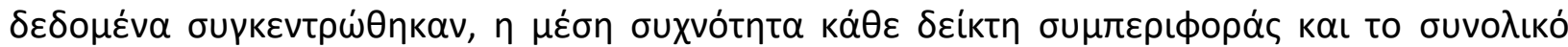

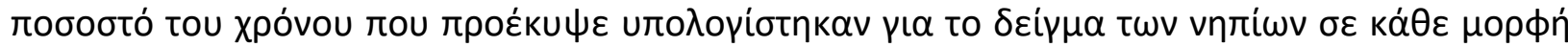

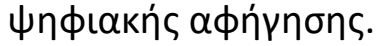

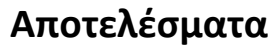

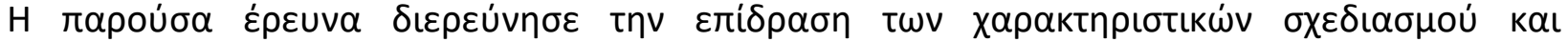

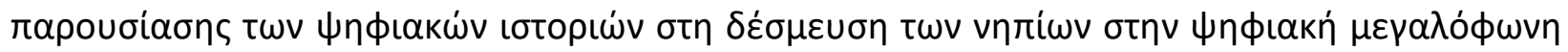

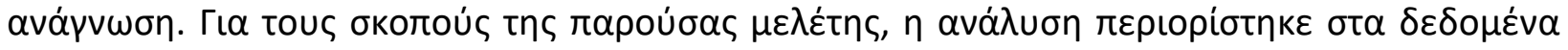

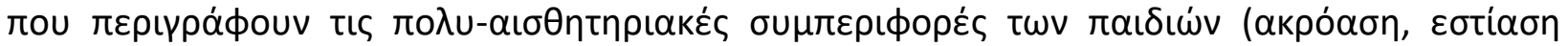




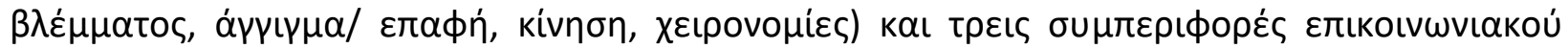

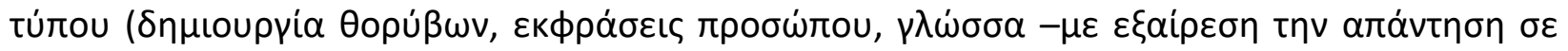

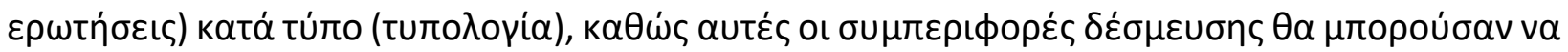

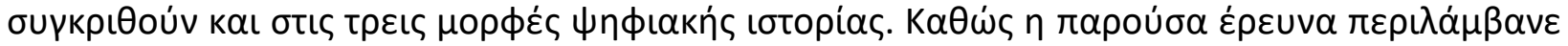

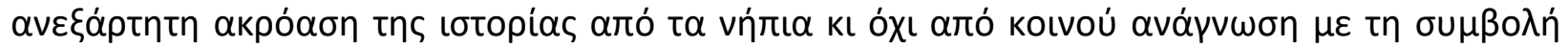

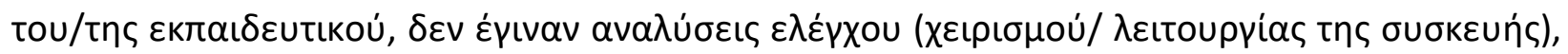

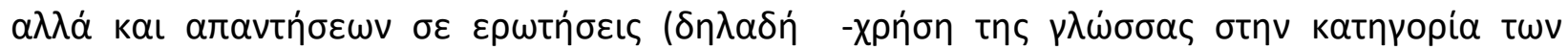

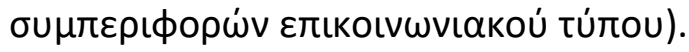

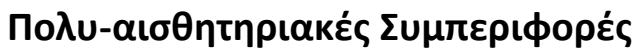

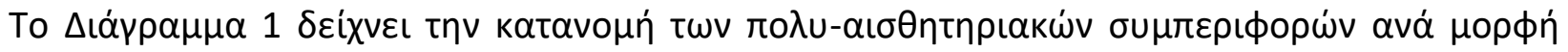

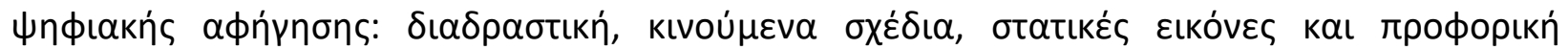

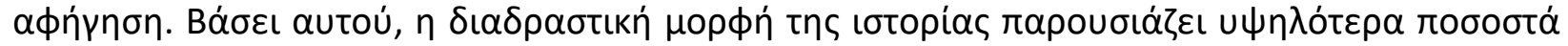

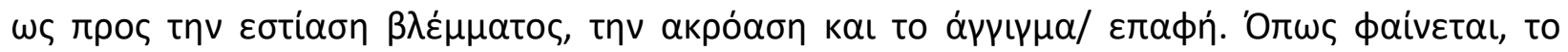

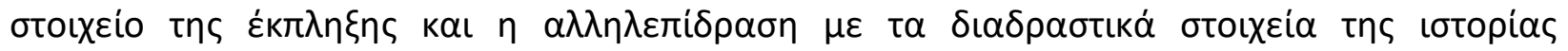

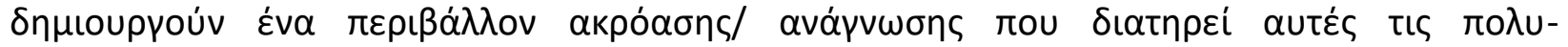

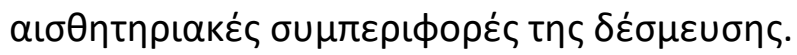

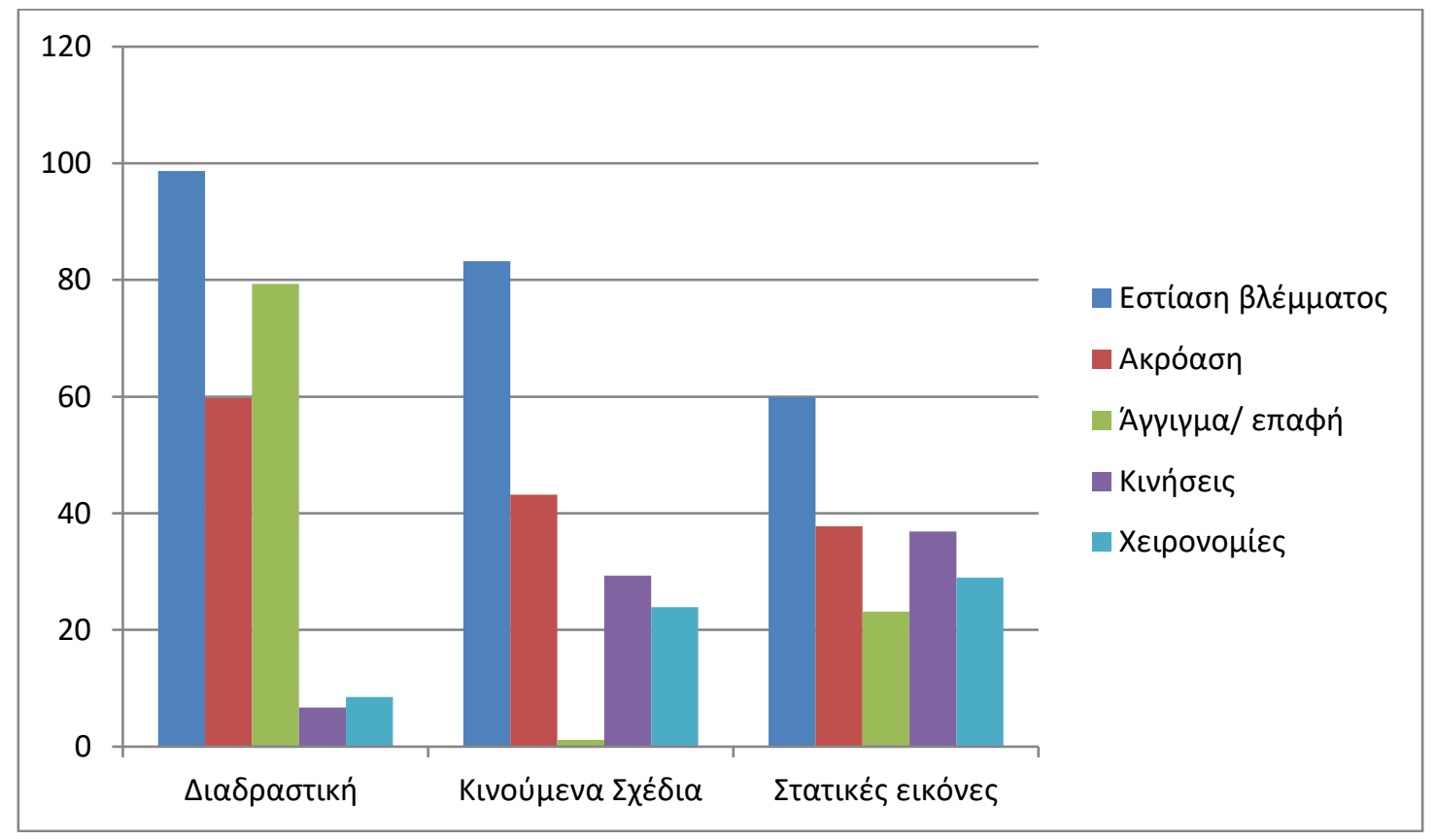

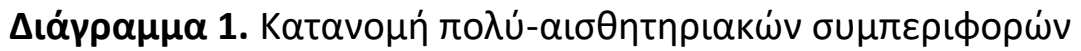

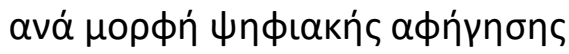

\begin{tabular}{|c|c|c|c|}
\hline & 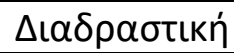 & 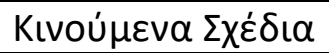 & 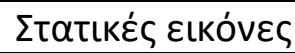 \\
\hline 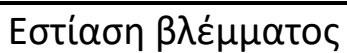 & $98.67 \%$ & $83.25 \%$ & $59.93 \%$ \\
\hline Акро́ $\alpha$ бn & $59.76 \%$ & $43.21 \%$ & $37.82 \%$ \\
\hline 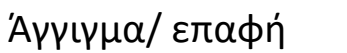 & $79.34 \%$ & $1.17 \%$ & $23.15 \%$ \\
\hline 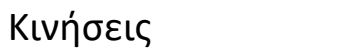 & $6.71 \%$ & $29.31 \%$ & $36.87 \%$ \\
\hline 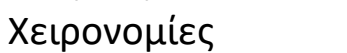 & $8.53 \%$ & $23.9 \%$ & $28.97 \%$ \\
\hline
\end{tabular}


MULTILINGUAL ACADEMIC JOURNAL OF EDUCATION AND SOCIAL SCIENCES

Vol. 8 No. 1, 2020, E-ISSN: 2308-0876 @ 2020 KWP

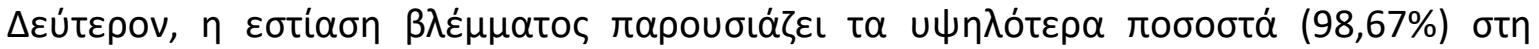

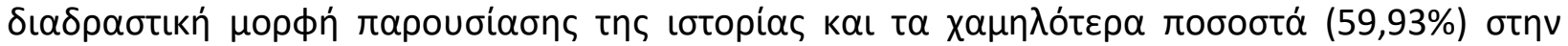

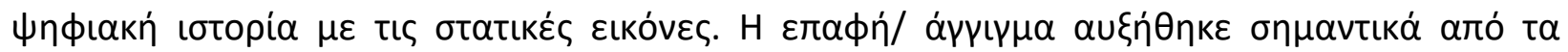

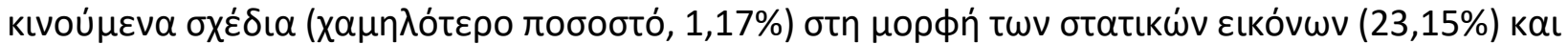

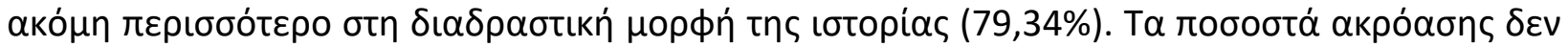

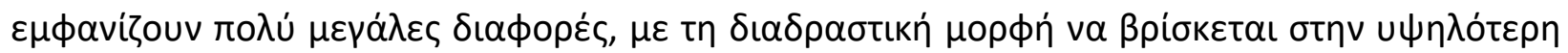

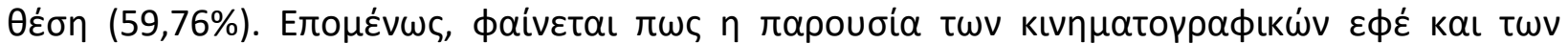

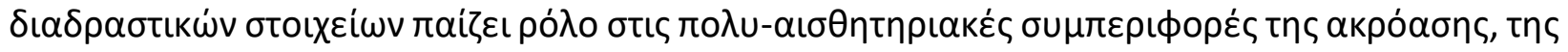

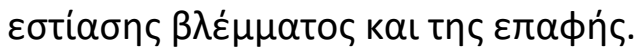

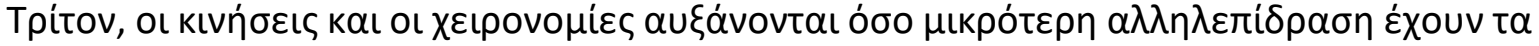

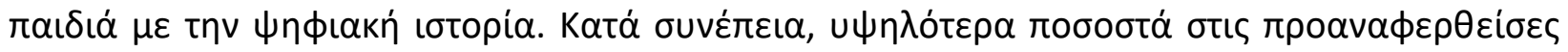

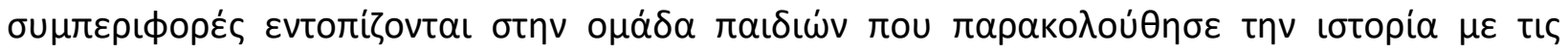

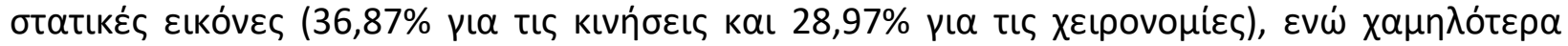

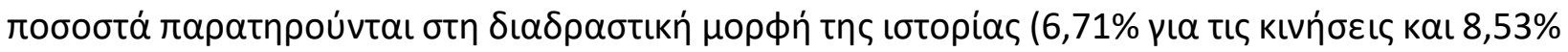

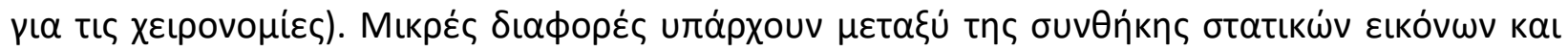

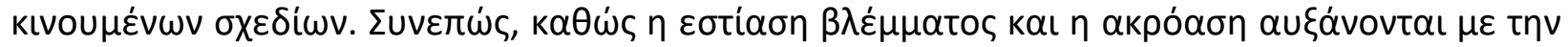

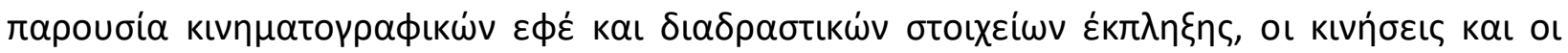

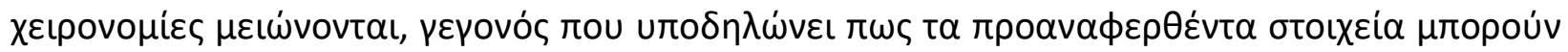

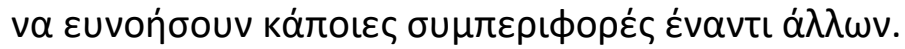

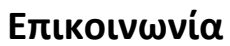

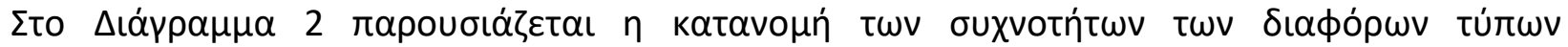

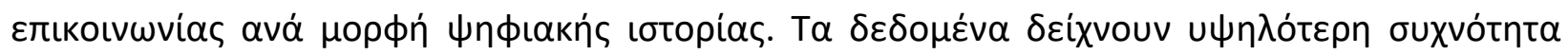

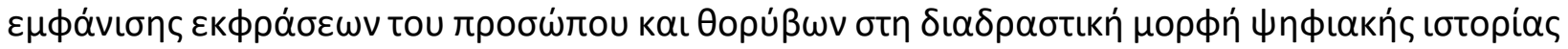

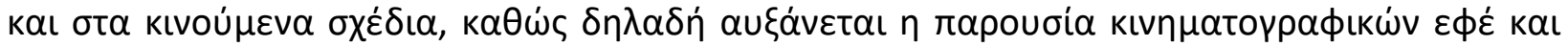

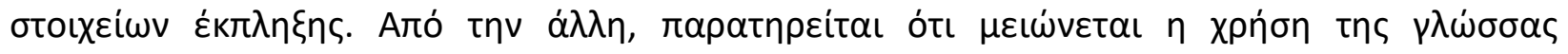

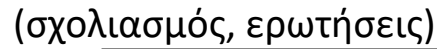

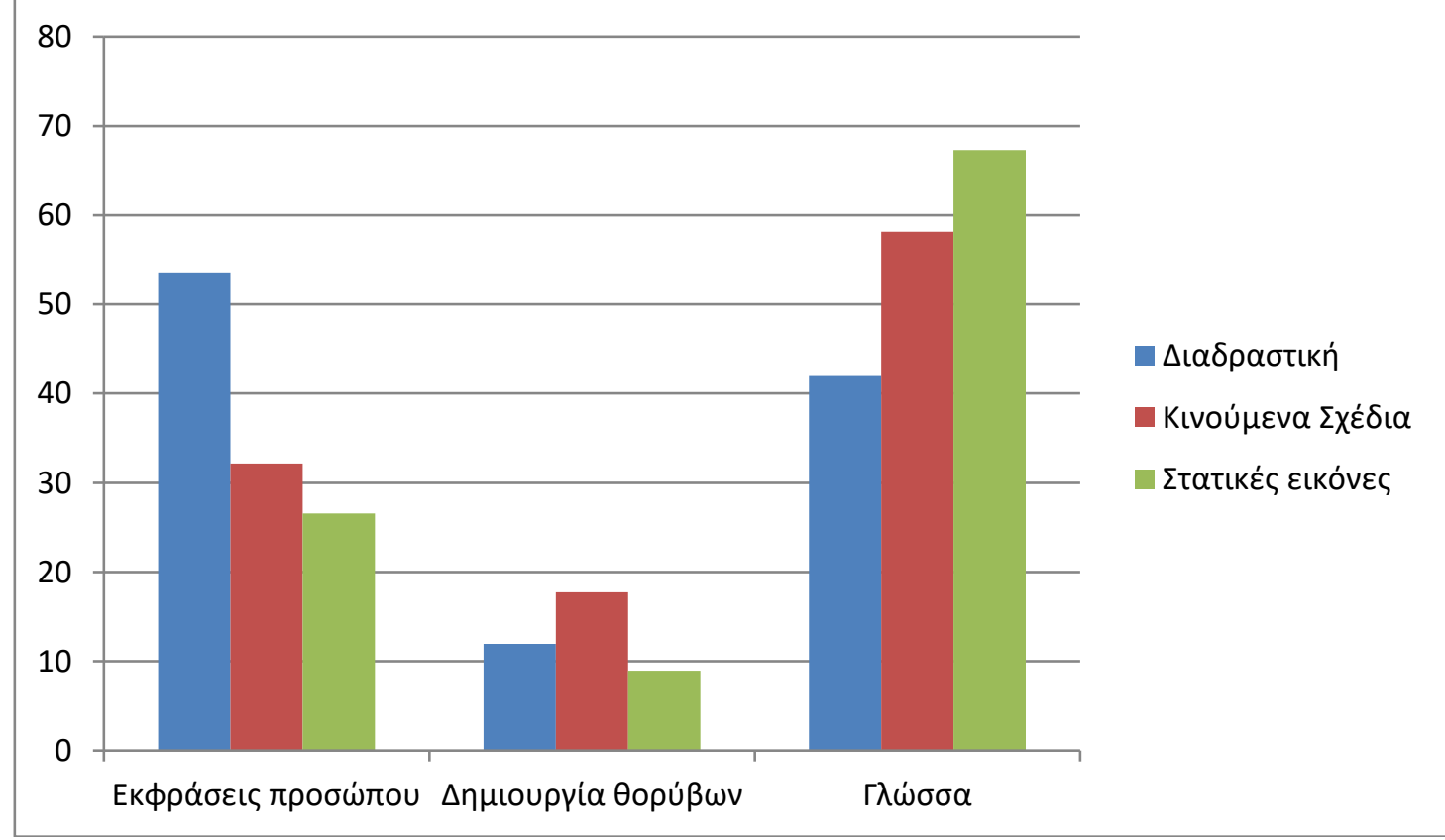


MULTILINGUAL ACADEMIC JOURNAL OF EDUCATION AND SOCIAL SCIENCES

Vol. 8 No. 1, 2020, E-ISSN: 2308-0876 @ 2020 KWP

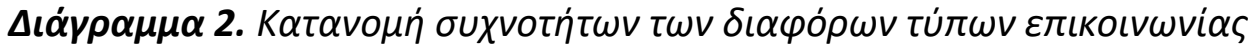

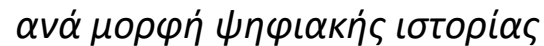

\begin{tabular}{|c|c|c|c|}
\hline & $\Delta \iota \alpha \delta \rho \alpha \sigma \tau \iota \kappa n ́$ & Kıvoú $\mu \varepsilon v \alpha \Sigma \chi \varepsilon \dot{\delta} \delta\llcorner\alpha$ & 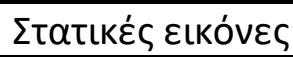 \\
\hline 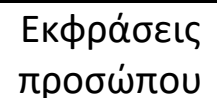 & $53.46 \%$ & $32.17 \%$ & $26.58 \%$ \\
\hline 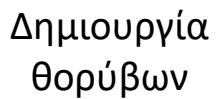 & $11.96 \%$ & $17.72 \%$ & $8.95 \%$ \\
\hline$\Gamma \lambda \omega \dot{\sigma} \sigma \sigma \alpha$ & $41.97 \%$ & $58.13 \%$ & $67.32 \%$ \\
\hline
\end{tabular}

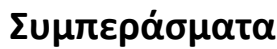

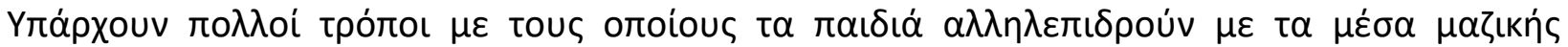

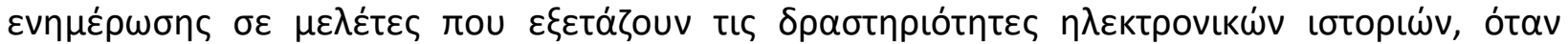

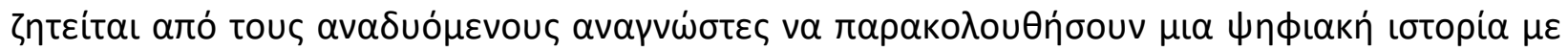

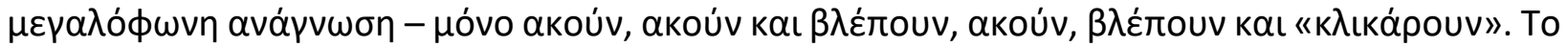

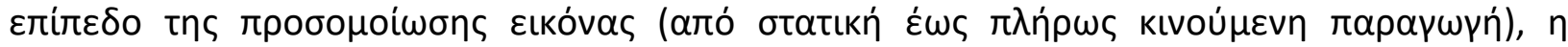

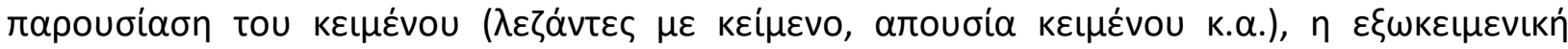

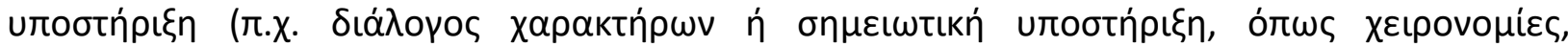

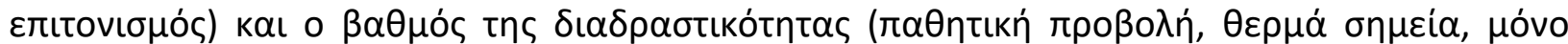

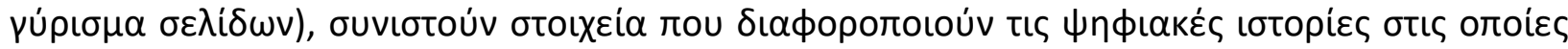

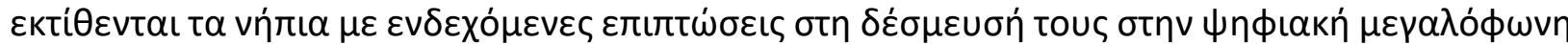

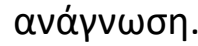

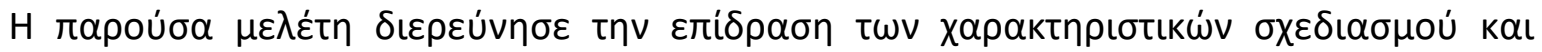

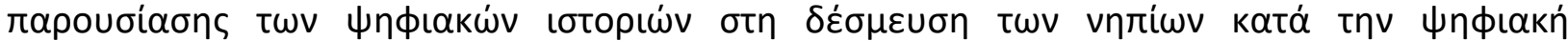

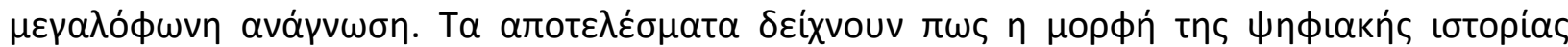

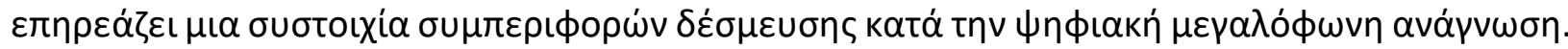

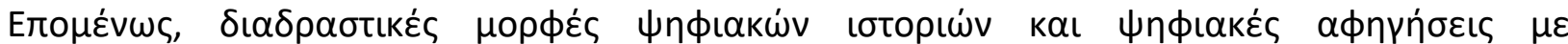

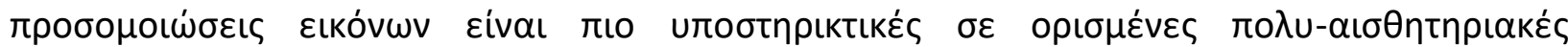

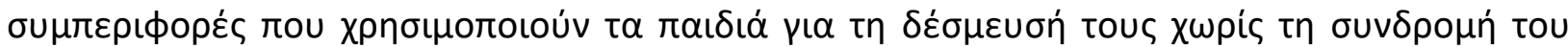

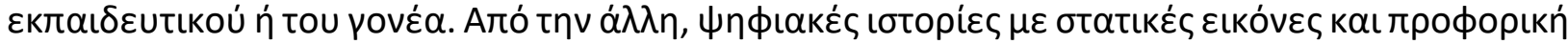

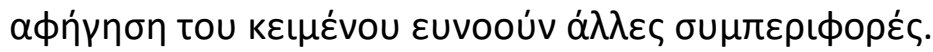

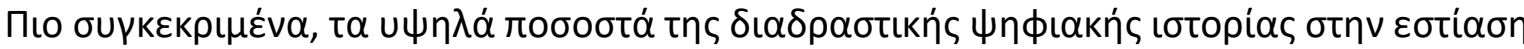

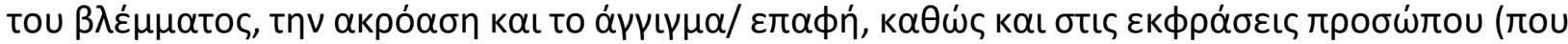

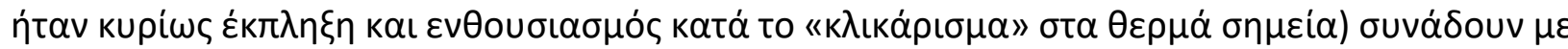

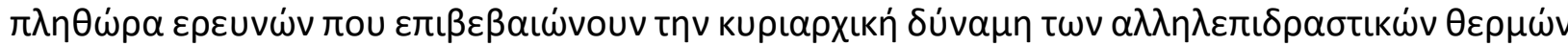

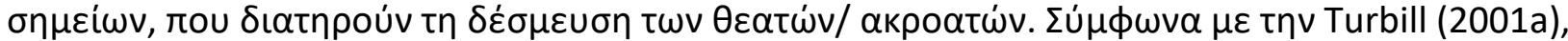

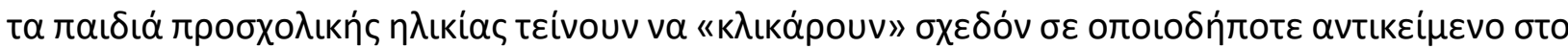

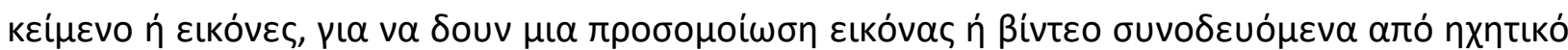

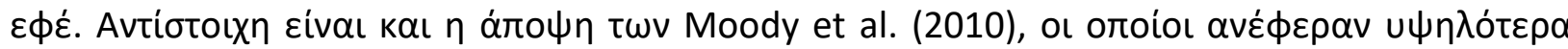

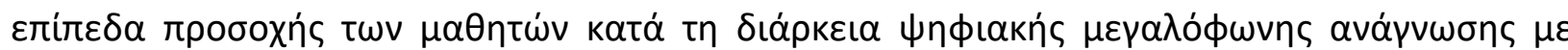

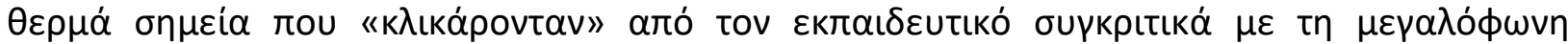

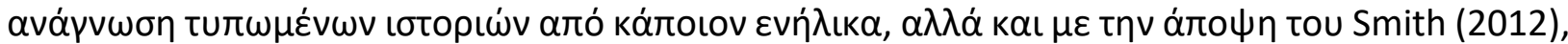

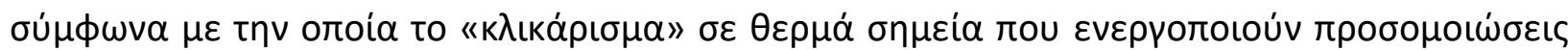

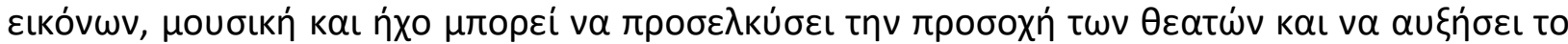

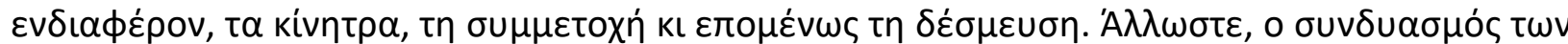


MULTILINGUAL ACADEMIC JOURNAL OF EDUCATION AND SOCIAL SCIENCES

Vol. 8 No. 1, 2020, E-ISSN: 2308-0876 @ 2020 KWP

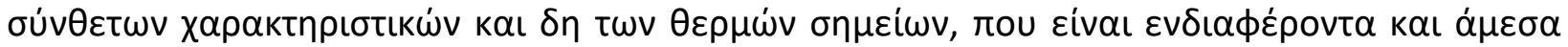

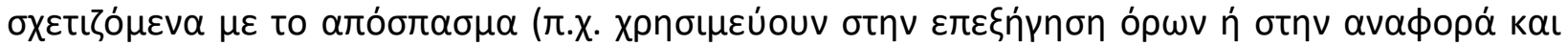

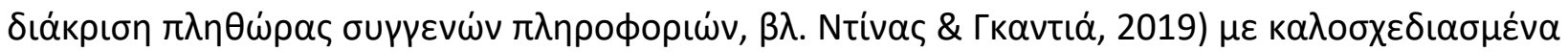

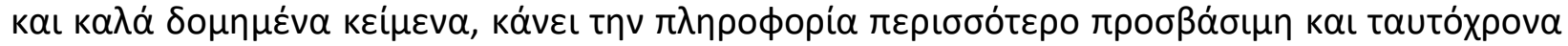

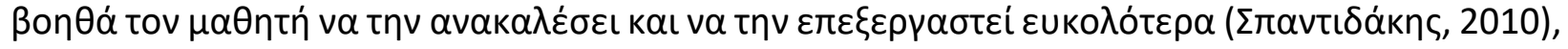

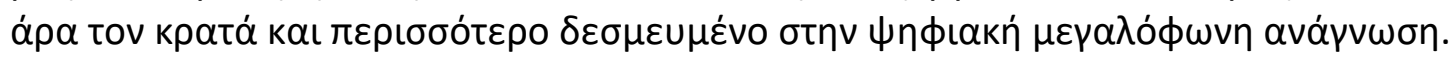

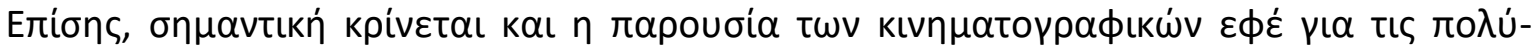

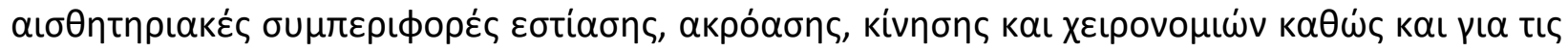

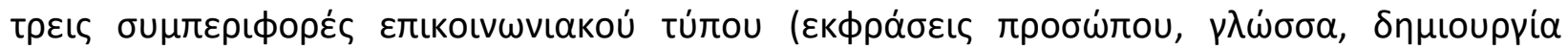

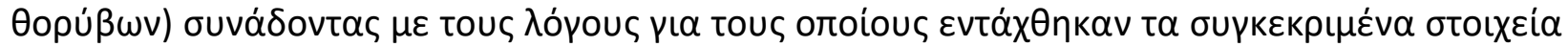

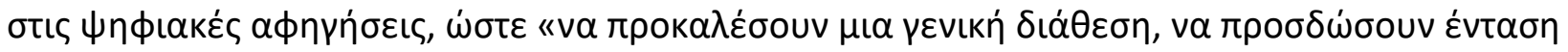

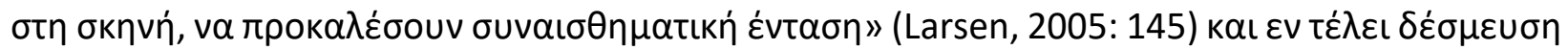

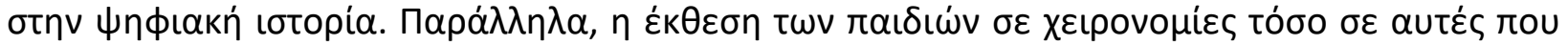

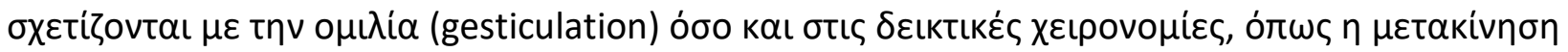

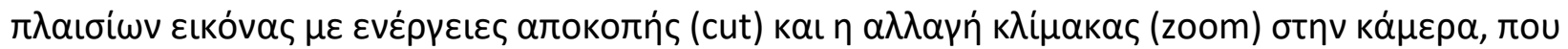

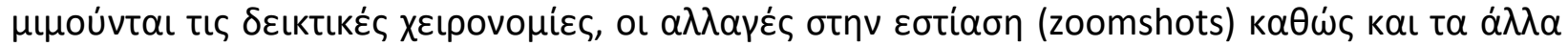

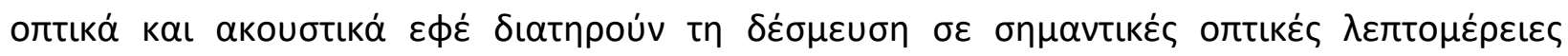

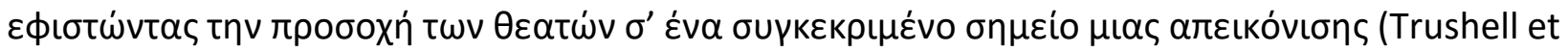

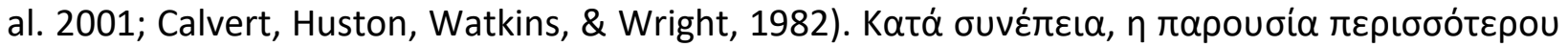

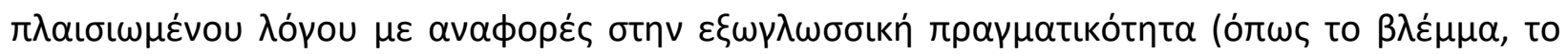

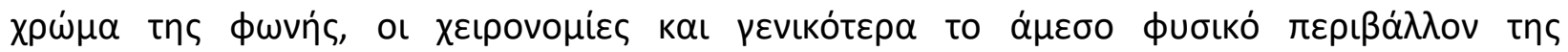

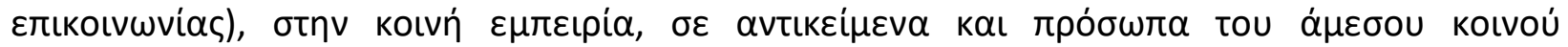

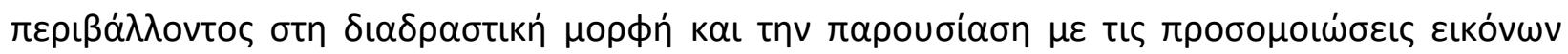

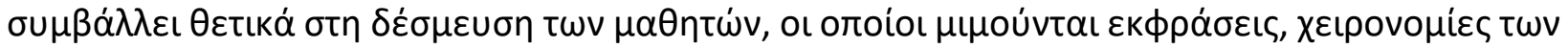

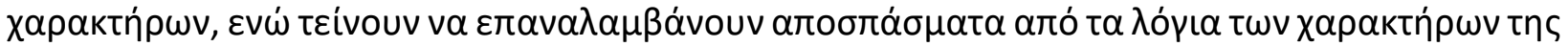

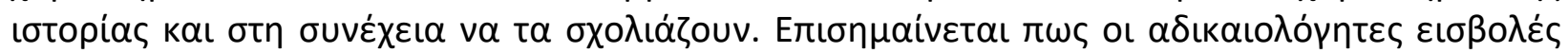

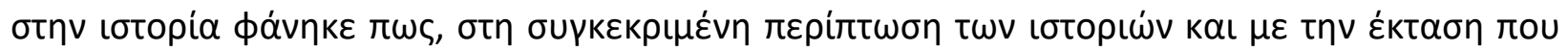

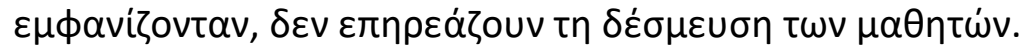

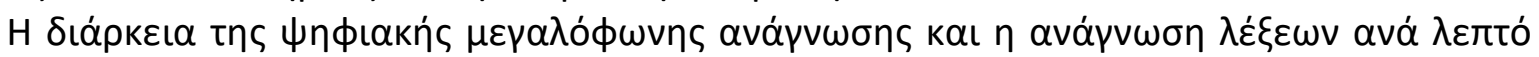

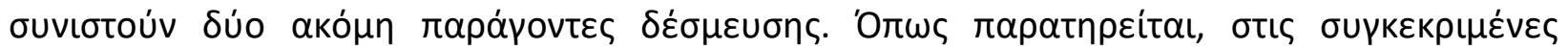

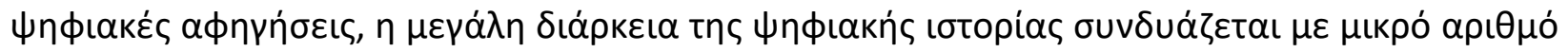

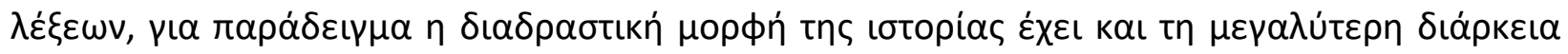

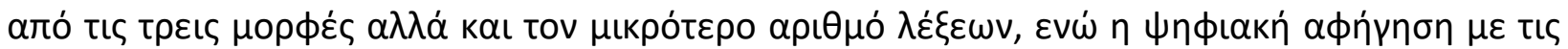

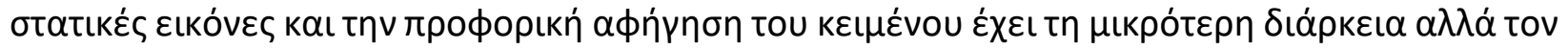

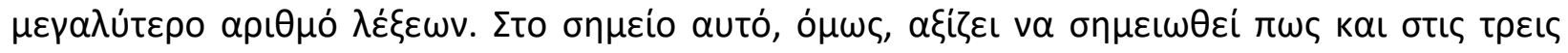

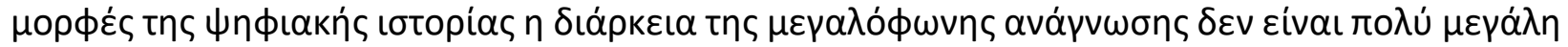

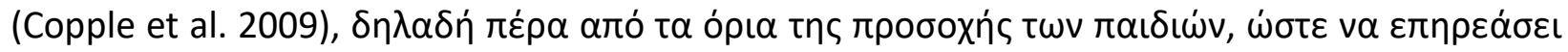

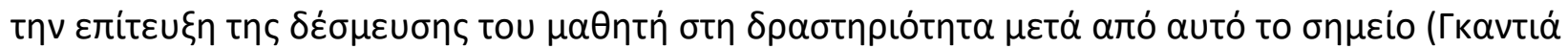

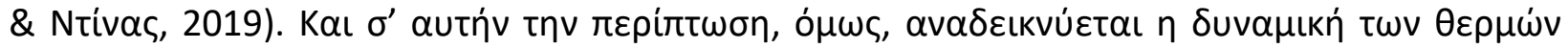

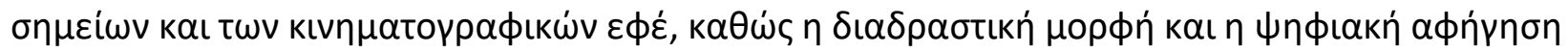

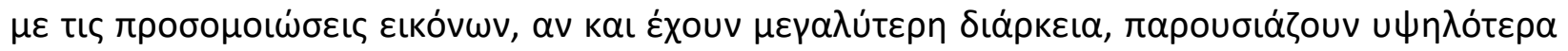

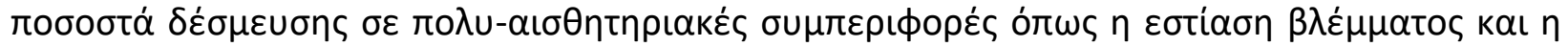

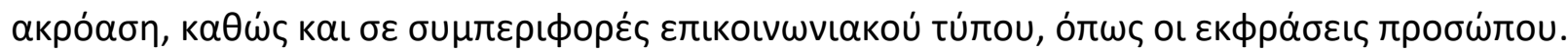


MULTILINGUAL ACADEMIC JOURNAL OF EDUCATION AND SOCIAL SCIENCES

Vol. 8 No. 1, 2020, E-ISSN: 2308-0876 @ 2020 KWP

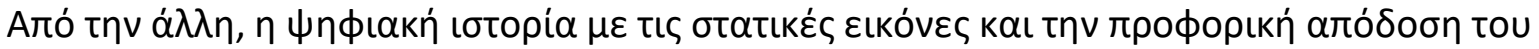

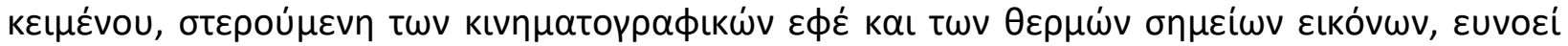

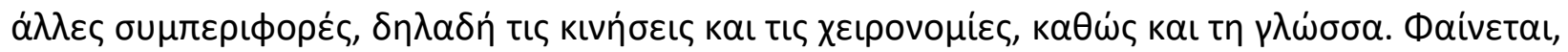

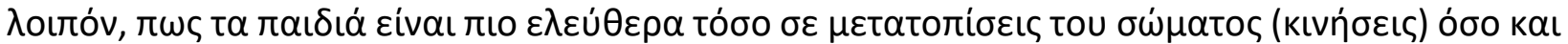

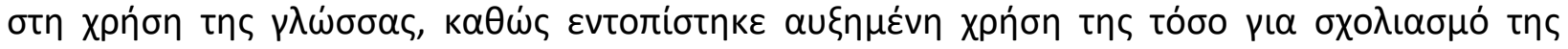

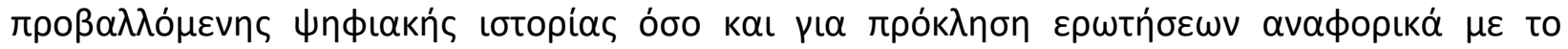

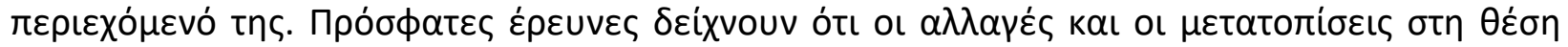

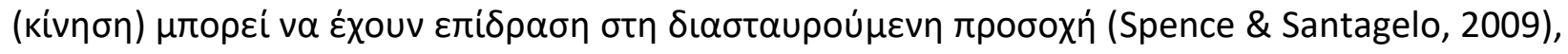

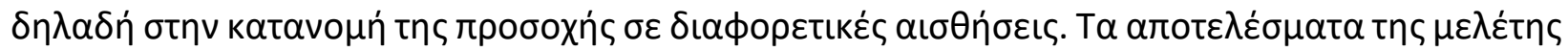

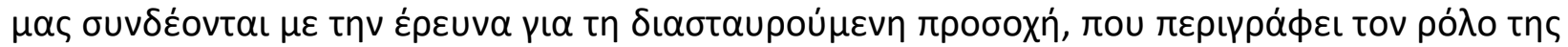

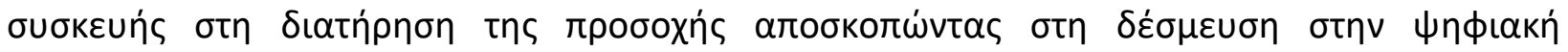

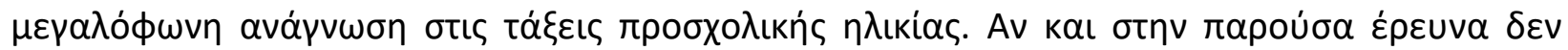

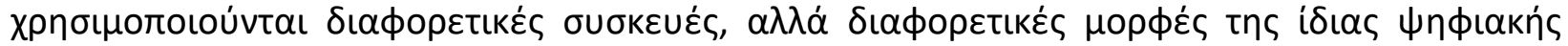

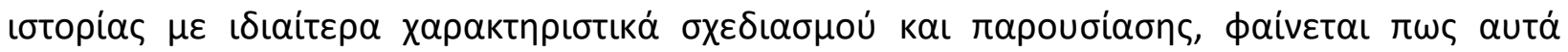

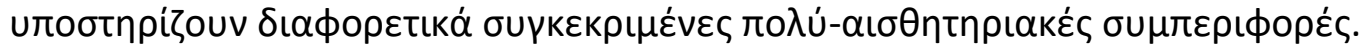

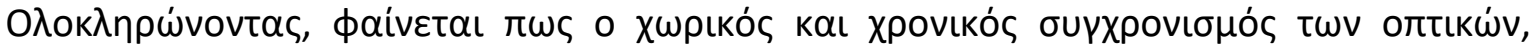

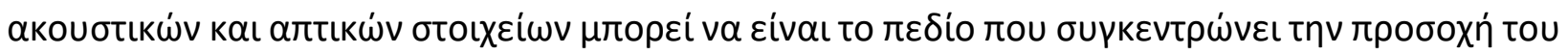

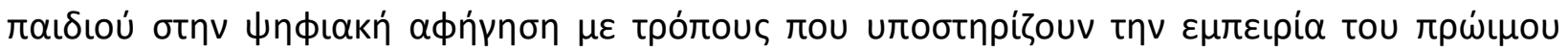

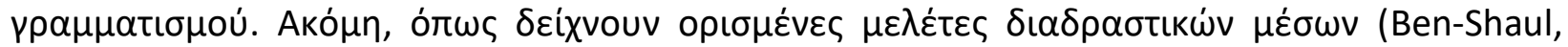

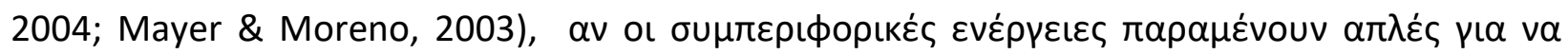

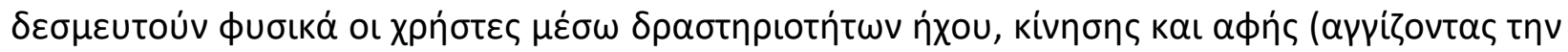

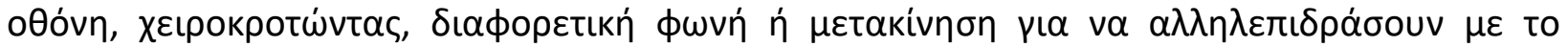

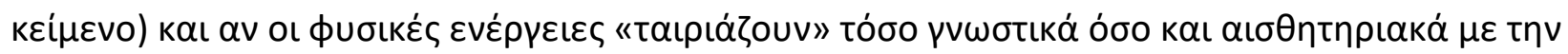

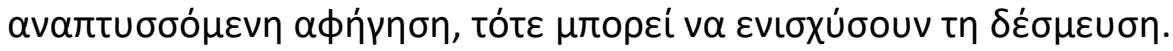

\section{References}

Barker, R. G. (1968). Ecological psychology: Concepts and methods for studying the environment of human behavior. Stanford, CA: Stanford University Press.

Ben-Shaul, N. (2004). Can narrative films go interactive? New Cinemas: Journal of Contemporary Film, 2, 149-162.

Bus, A., \& Van IJzendoorn, M. (1995). Mothers reading to their 3-year-olds: The role of motherchild attachment security in becoming literate. Reading Research Quarterly, 30, 9981015.

Calvert, S. L., Huston, A. C., Watkins, B. A., \& Wright, J. C. (1982).The relation between selective attention to television forms and children's comprehension of content. Child Development, 53, 601-610.

Chen, M., Ferdig, R., \& Wood, A. (2003). Understanding technology-enhanced storybooks and their roles in teaching and learning: An investigation of electronic storybooks in education. Journal of Literacy and Technology, 3(1) .

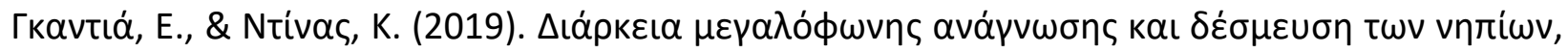

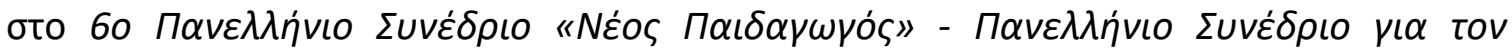

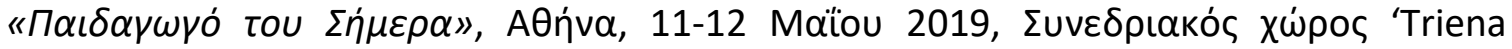
Business Center'. 
MULTILINGUAL ACADEMIC JOURNAL OF EDUCATION AND SOCIAL SCIENCES

Vol. 8 No. 1, 2020, E-ISSN: 2308-0876 @ 2020 KWP

Connor, C. M., Jakobsons, L. J., Crowe, E., \& Meadows, J. (2009). Instruction, differentiation, and student engagement in Reading First classrooms. Elementary School Journal, 109, 221250.

Csikszentmihaly, M, (1996). Creativity: Flow and the psychology of discovery and invention. New York: Harper Collins.

Cummings, K. D., Otterstedt, J., Kennedy, P. C., Baker, S. K., \& Kame'enui, E. J. (2011). DIBELS Data System: 2009-2010 Percentile Ranks for DIBELS 6th Edition Benchmark Assessments (Technical Report 1102).Eugene, OR: University of Oregon, Center on Teaching and Learning. Available https://dibels.uoregon.edu/research/techreports/\#dibels

De Jong, M.T., \& Bus, A. G. (2003). How well suited are electronic books to supporting literacy? Journal of Early Childhood Literacy, 3(2), 147-164.

FinN, J. D., Pannozzo, G. M., \& Voelkl, K. E. (1995). Disruptive and inattentive - withdrawn behavior and achievement among fourth graders. Elementary School Journal, 95, (421454).

Guthrie, J. T., \& Wigfield, A. (2000). Engagement and motivation in reading. In P. D. Pearson, R. Barr, M. Kamil \& P. Mosenthal (Eds.), Handbook of reading research (Vol. III, pp. 403-422). Mahaw, NJ: Erlbaum.

Greeno, J. (1994). Gibson's affordances. Psychological Review, 101, 336-342.

Jago, C. (2005). Papers, papers, papers: An English Teacher's Survival Guide. Portsmouth, NH: Heinemann.

Javorsky, K., \&Trainin, G. (2014). Teaching young readers to navigate a digital story when rules keep changing. The Reading Teacher .

Korat, O., \& Shamir, A. (2004). Do hebrew electronic books differ from Dutch electronic books? A replication of a Dutch content analysis. Journal of Computer Assisted Learning, 20, 257268.

Larsen, P. (2005). Filmmusikk. Oslo: Universitetsforlaget.

Mayer, R., \& Moreno, R. (2003). Nine ways to reduce cognitive load in multimedia learning. Educational Psychologist, 38, 43-52.

Moody, A., Justice, L., \& Cabell, S. (2010). Electronic versus traditional storybooks: Relative influence on preschool children's engagement and communication. Journal of Early Childhood Literacy, 10 (3), 294-313.

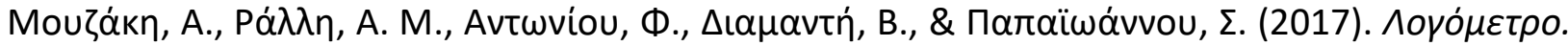

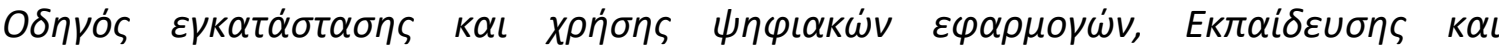

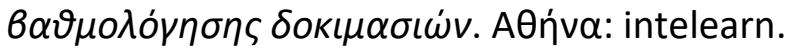

New London Group. (1996). A Pedagogy of Multiliteracies: Designing Social Futures. Harvard Educational Review, 66, 60-92.

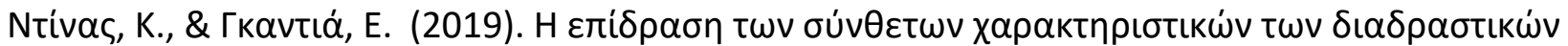

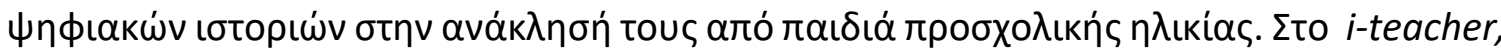

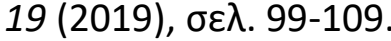

Pressley, M., Wharton-McDonald, R., Mistretta-Hampston, J. M., \& Echevarria, M. (1998). The nature of literacy instruction in ten grade $4 / 5$ classrooms in upstate New York. Scientific Studies of Reading, 2, 159-194.

Reinking, D. (Ed.). (1998). Handbook of literacy and technology: Transformations in a posttypographic world. Mahwah, NJ: Erlbaum. 
MULTILINGUAL ACADEMIC JOURNAL OF EDUCATION AND SOCIAL SCIENCES

Vol. 8 No. 1, 2020, E-ISSN: 2308-0876 @ 2020 KWP

Reinking, D., McKenna, M. C., Labbo, L. D., \& Kieffer, R. D. (1998). Handbook of literacy and technology: Transformations in a post-typographic world. Mahwah, NJ: Erlbaum.

Roskos, K., Burstein, K., You, B.-K., Brueck, J., \& O’Brien, C. (2011). A formative study of an e-book instructional model in early literacy. Creative Education, 2, 10-17.

Roskos, K., \& Brueck, J. (2011). Developing an e-book quality rating tool. Paper presented at the annual meeting of the American Educational Research Association, New Orleans, LA.

Roskos, K., Burstein, K., \& You, B.-K. (2012). A typology for observing children's engagement with ebooks at preschool. Journal of Interactive Online Learning, 11, 47-66.

Samuels, S. J., \&Turnure, J. E. (1974). Attention and reading achievement in firstgrade boys and girls. Journal of Educational Psychology, 66(1), 29-32.

Sipe, L. (2002). Talking back and taking over: Young children's expressive engagement during storybook read-alouds. The Reading Teacher, 55(45), 467- 483.

Smith, P. K. (2010). Children and play. Chichester, West Sussex, UK: Wiley-Blackwell.

Smith, B. E. (2013). Composing across modes: Urban adolescents' processes responding to and analyzing literature. Dissertation. Vanderbilt University. Nashville, TN.

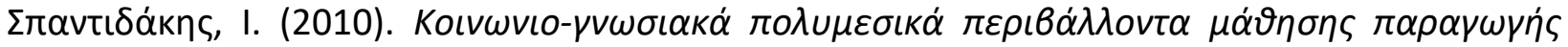

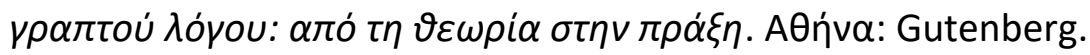

Spence, C., \& Santangelo, V. (2009). Capturing spatial attention with multisensory cues. Hearing Research, 258, 134-142.

Teale, W., \& Sulzby, E. (1986). Emergent literacy as a perspective for examining how young children become writers and readers. Introduction in Emergent literacy: Writing and reading. Norwood, NJ: Ablex Publishing Corporation.

Tierney, R. J. (2008). Learning with multiple literacies: observations of lives exploring meanings, identities, possibilities, and worlds. In J. Flood, S. B. Heath, \& D. Lapp (Eds.). Handbook of Research on Teaching Literacy Through the Communicative and Visual Arts Volume II (pp. 101-108). New York: Taylor \& Francis Group.

Trushell, J., Burrell, C., \& Maitland, A. (2001). Year 5 pupils reading an 'Interactive Storybook' on CD ROM: Losing the plot? British Journal of Educational

Technology, 32(4), 389-401.

Turbill, J. (2001a). A researcher goes to school: Using technology in the kindergarten literacy curriculum. Journal of Early Childhood Literacy, 1(3), 255-279.

Unsworth, L. (2003). Reframing research and literacy pedagogy relating to CD narratives: Addressing 'radical change' in digital age literature for children. Issues in Educational Research, 13.

Van den Broek, P., Kendeou, P., \& White, M. J. (2009). Cognitive processes during reading:Implications for the use of multimedia to foster reading comprehension. In A.G. Bus \&S.B. Neuman (Eds.), Multimedia and literacy development (pp. 57- 74). New York:Routledge.

Verhallen, M., Bus, A. G., \& de Jong, M. T. (2006). The promise of multimedia stories for Kindergarten children at risk. Journal of Educational Psychology, 98(2), 410-419.

Vygotsky, L. (1978). Mind in society. Cambridge, MA: Harvard University Press.

Yaden, D. B., Rowe, D. W., \& MacGillivray, L. (2000). Emergent literacy: A Matter(Polyphony) of Perspectives. M. Kamil, P. Mosenthal, D. Pearson, \& R. Barr (Eds.), Handbook of reading research. V III (pp. 425-454). Mahwah, NJ: Lawrence Erlbaum. 